\title{
Cooperation between Caenorhabditis elegans COMPASS and condensin in germline chromatin organization
}

\section{Marion Herbette}

Ecole Normale Superieure de Lyon, Université de Lyon

\section{Valérie Robert}

Ecole Normale Superieure de Lyon, Université de Lyon

Aymeric Bailly

CRBM, Univeristy of Montpellier

\section{Loïc Gely}

Ecole Normale Supérieure de Lyon

\section{Robert Feil}

Institute of Molecular Genetics of Montpellier, Univerisity of Montpellier

\section{David Llères}

Universite de Montpellier

Francesca Palladino ( $\nabla$ francesca.palladino@ens-lyon.fr)

Ecole normale superieure de Lyon https://orcid.org/0000-0003-3087-449X

\section{Research}

Keywords: COMPASS, SET1, FLIM-FRET, condensin, topoisomerase, C. elegans, meiosis

Posted Date: March 17th, 2020

DOl: https://doi.org/10.21203/rs.3.rs-17459/v1

License: (c) (i) This work is licensed under a Creative Commons Attribution 4.0 International License. Read Full License 
1 Cooperation between Caenorhabditis elegans COMPASS and condensin in germline chromatin

2 organization

3 M. Herbette1, V. Robert, A. Bailly2, L. Gely1, R. Feil3, D. Llères3 and F. Palladino1,4

4 1Laboratory of Biology and Modeling of the Cell, UMR5239 CNRS/Ecole Normale Supérieure de Lyon,

5 INSERM U1210, UMS 3444 Biosciences Lyon Gerland, Université de Lyon, Lyon, France.

$6 \quad$ 2CRBM, University of Montpellier, CNRS, France.

7 3Institute of Molecular Genetics of Montpellier (IGMM), CNRS, University of Montpellier,

8 Montpellier, France.

94 corresponding author

11 Abstract

Background

13 Histone-modifying activities play important roles in gene expression and influence higher-order 14 genome organization. SET1/COMPASS (Complex Proteins Associated with Set1) deposits histone $\mathrm{H} 3$ lysine 4 (H3K4) methylation at promoter regions and is associated with context-dependent effects on gene expression. Whether it also contributes to higher-order chromosome organization has not been explored.

Results

Using a quantitative FRET (Förster resonance energy transfer)-based fluorescence lifetime imaging microscopy (FLIM) approach to assay nanometer scale chromatin compaction in live animals, we reveal

21 a novel role for SET1/COMPASS in structuring meiotic chromosomes in the C. elegans germline. Inactivation of SET-2, the C. elegans homologue of SET1, strongly enhanced chromosome organization defects and loss of fertility resulting from depletion of condensin-II, and aggravated defects in chromosome morphology resulting from inactivation of topoisomerase II, another major structural component of chromosomes. Loss of CFP-1, the chromatin targeting subunit of COMPASS, similarly

26 affected germline chromatin compaction measured by FLIM-FRET and enhanced condensin-II knock27 down phenotypes. 


\section{Conclusions}

29 The data presented here are consistent with a role of SET1/COMPASS in shaping meiotic chromosomes

30 in the C. elegans germline. This new insight has important implications for how chromatin-modifying

31 complexes and histone modifications may cooperate with non histone-proteins to achieve proper

32 chromosome organization, not only in meiosis, but also in mitosis.

33

34 COMPASS, SET1, FLIM-FRET, condensin, topoisomerase, C. elegans, meiosis

35 


\section{Background}

In different species from yeast to mammals, chromatin modifying complexes and histone posttranslational modifications (PTMs) contribute to higher-order chromatin structure (1). While the spatial configuration of chromatin has been shown to be essential to ensure fundamental processes from gene expression to cell divisions, how such higher-order structures are formed in various cellular processes remains unclear. Mitosis and meiosis, two essential processes that require restructuring and reorganization of chromatin architecture, are associated with specific changes in histone modifications. During mitosis, extensive compaction of chromatin is associated with histone H3 serine 10 phosphorylation (H3S10ph), H4K20 monomethylation (H4K20me1), and a dramatic reduction in overall histone acetylation (2-4). Specific histone PTMs, including H3K4 tri-methylation (H3K4me3), are also associated with meiotic double strand breaks (DSBs) during recombination, and are dynamically altered during meiotic progression (5-8).

The SET1 class of histone methyltransferases act in large multi-subunit complexes known as COMPASS $(9,10)$ to deposit $\mathrm{H} 3 \mathrm{~K} 4 \mathrm{me} 3$ at promoters of actively transcribed genes. At promoters, levels of COMPASS-dependent H3K4 methylation correlate with transcription levels, but evidence for an instructive role for $\mathrm{H} 3 \mathrm{~K} 4 \mathrm{me} 3$ in transcription is lacking, and recent data suggest that its role depends on different chromatin and cellular contexts (11).

One well-characterized function of $\mathrm{H} 3 \mathrm{~K} 4$ methylation relies on the binding of effector proteins to mediate downstream processes related to transcription (12-15). Recent data suggest that $\mathrm{H} 3 \mathrm{~K} 4 \mathrm{me} 3$ may also play a more direct role in specific aspects of chromatin organization. For instance, genomewide changes in H3K4 methylation are observed during mammalian spermatogenesis (16), with a large fraction of dynamic $\mathrm{H} 3 \mathrm{~K} 4 \mathrm{me} 3$ not coinciding with gene promoters or double strand break (DSB). In mouse oocytes, H3K4 methylation defines distinct nanoscale compartments associated with active transcription that contribute to the shaping of meiotic chromosomes (17).

Additional evidence linking SET1/COMPASS to higher-order chromosome organization comes from studies of individual subunits of the complex. For example, in mouse absence of the COMPASS targeting subunit CFP1 results in defects in meiotic oocyte maturation, defective spindle assembly and chromosome misalignment, with only minor effects on transcription (18). In fission yeast, SET1 plays 
both H3K4-dependent and -independent roles in genome organization through long-range clustering of retrotransposon loci (19-21).

C. elegans contains a single SET1 homolog encoded by set-2 $(22,23)$. set-2 inactivation results in defective patterns of $\mathrm{H} 3 \mathrm{~K} 4 \mathrm{me} 3$ in the germline and progressive misregulation of the germline transcriptome, leading to increased genome instability and sterility (22-25). Whether these effects reflect a direct role in transcription, or a more general role in germline chromatin organization is not known. Significantly, we previously found no correlation between COMPASS dependent H3K4me3 at promoters and transcription in C. elegans embryos, consistent with recent observations in other organisms (26-30). Likewise, increased genome instability in set-2 mutant germlines was not associated with defects in the induction of the DNA damage response (DDR) pathway, suggesting downstream effects in the DNA repair process (24).

In this study, we used fluorescence lifetime imaging microscopy (FLIM) for Förster Resonance Energy Transfer (FRET) measurements in live animals to assess close proximity between nucleosomes and to quantify different levels of chromatin compaction. In meiotic cells from animals lacking COMPASS components SET-2 and CFP-1, we find that FRET between fluorophore-tagged nucleosomes is dramatically decreased, consistent with a structural role for COMPASS affecting nucleosome proximity in the nucleus. In support of such a role, we find that loss of either set-2 or $c f p-1$ enhances the chromosome organization defects resulting from reduced expression of condensin-II, a major contributor to chromosome structure (31). Cooperation between SET-2 and condensin-II in the germline was independently confirmed in animals carrying a temperature-sensitive allele of the condensin-II subunit $h c p-6$. We further show that set-2 inactivation aggravates the germline phenotypes resulting from conditional inactivation of topoisomerase II, another major component of chromosomes (32). Altogether, our results indicate that COMPASS contributes to the organization of germline chromosome architecture in cooperation with condensin, and have wider implications on the role of COMPASS-related complexes and H3K4 methylation in higher-order chromatin structure.

\section{Results}

\section{Nanoscale chromatin compaction is decreased in set-2 mutant germlines}


In the $C$. elegans germline, meiotic nuclei are arranged in a temporal-spatial order, with the distal end of the gonad containing mitotically proliferating nuclei. Homolog pairing initiates downstream in the "transition zone", followed by pachytene, during which synapsed chromosomes appear in DAPI-stained nuclei as discrete, parallel tracks. More proximally, nuclei exit pachytene, enter diplotene, and cellularized oocytes containing condensed homologs are formed (33).

$\mathrm{H} 3 \mathrm{~K} 4 \mathrm{me} 3$ is detected on chromatin in all germline nuclei, from the distal mitotic region through the meiotic stages and into diakinesis (Figure S1A)(22,23). In germlines from animals carrying the set2(bn129) loss-of-function frameshift allele that results in a premature stop codon (22), H3K4me3 strongly decreases in the distal mitotic region through early-mid pachytene. Levels of H3K4me3 are not visibly altered in late pachytene and diakinetic nuclei of mutant animals, most likely reflecting the additional activity of SET-16/MLL, the only other SET1 family member in C. elegans (Figure S1A, $(22,23,34,35)$. Reduced H3K4 methylation in set-2(bn129) animals is associated with a progressive

104 decrease in fertility at the stressful temperature of $25^{\circ} \mathrm{C}$, resulting in sterility at the $\mathrm{F} 4-\mathrm{F} 5$ generation 105 (22,23). These observations suggest that H3K4 methylation, or SET 2 itself, play an important role in 106 germline maintenance. DAPI staining of chromatin revealed no apparent defects in either germline 107 organization, or chromosome morphology in set-2 mutant animals raised under normal conditions $108\left(20^{\circ} \mathrm{C}\right)$, or late generation fertile animals at $25^{\circ} \mathrm{C}$ (data not shown and (22). Interestingly however, in late generation (F4) germline nuclei from animals approaching sterility at $25^{\circ} \mathrm{C}$, chromatin appeared less organized than in wildtype animals, with a loss of the distinctive pachytene nuclei morphology

111 (Figure S1B). These results suggest that SET-2 may have an impact on global genome organization.

112 Chromatin modifying complexes and histone modifications define the different functional states

113 of chromatin throughout the genome, and these in turn contribute to higher-order chromatin organization

114 (36). To investigate whether and how COMPASS influences higher-order chromatin compaction 115 specifically in germline cells, we used a recently developed FLIM-FRET technique that enables 116 quantification of chromatin condensation levels in live animals at the level of nucleosome packaging.

117 The assay is based on the measurement of FRET interaction between fluorescently-labelled core histone 118 GFP::H2B (donor) and mCherry::H2B (acceptor) co-expressed in the C. elegans germline (37). 119 Importantly, FRET is a phenomenon sensitive to fluorophore proximity that occurs efficiently only when 
120 the donor and acceptor fluorescent fusion proteins are closely positioned $(<10 \mathrm{~nm})$ in the 3D nuclear

121 space following chromatin compaction. By measuring histone-histone proximity, this FRET assay can

122 respond to changes in nucleosome spacing, and therefore provides a read-out of nanoscale chromatin

123 compaction (38-41). FLIM-FRET also provides accurate quantification due to the independence of the

124 fluorescence lifetime from the relative concentrations of the interacting proteins, and is independent of

125 their diffusion rates (42). To carry out the assay, we first generated wildtype and set-2 mutant strains

126 that stably co-express both GFP-H2B and mCherry-H2B fusion proteins (FPs, named "H2B-2FPs"

127 hereafter) from a single transcription unit driven by the germline-specific pmex-5 promoter (Figure 1A).

128 We confirmed that there was no alteration in the expression of fluorescent-tagged H2B histones in set-

1292 mutants (Figure S2A), and fluorescence recovery after photo-bleaching (FRAP) showed that tagged

130 histones H2B were stably incorporated into chromatin in set-2 mutants (Figure S2B).

131 Comparative FLIM-FRET analysis of set-2(bn129)H2B-2FPs and wtH2B-2FPs pachytene nuclei

132 revealed a strong reduction in chromatin compaction levels in the absence of set-2, as indicated by a

133 longer GFP-H2B fluorescence lifetime (Figure 1A), and a reduced mean-FRET efficiency (Figure 1B).

134 Quantification of FRET efficiencies in individual nuclei allowed us to arbitrarily define several classes

135 of FRET, from "sub-low FRET" to "high-FRET", as previously described (43). Interestingly, we

136 observed that in the absence of set-2, "intermediate-FRET" and "high-FRET" populations previously

137 linked to heterochromatic states (43) were significantly reduced compared to wild type (Figure 1C),

138 while the "sub-low-FRET" chromatin class associated with more accessible chromatin was increased.

139 These results suggest that the absence of SET-2 influences nanoscale chromatin structure in the

140 germline.

141 Loss of set-2 enhances chromosome organization defects resulting from condensin-II knock-down

142 If SET-2 contributes to chromatin organization in germ cells, one prediction is that its absence might

143 impact chromosome function and result in chromosome segregation defects in meiosis, thereby

144 generating aneuploid cells (44). We found no evidence for such defects in set-2 mutant animals (24, 30,

145 45). What we did find, however, is that endoreplicated intestinal cells of adult animals showed

146 chromosome segregation defects very similar to those reported in condensin-II mutants $(45,46)$. This

147 raised the possibility that in normally dividing cells of these mutants, more subtle defects in germline 
148 chromatin organization arise that become clearly apparent only in a sensitized background in which

149 chromatin structure is further perturbed.

150 Condensins are major contributors to chromosome structure and organization (31). Metazoans

151 contain two types of condensin complexes (I and II) that share a heterodimer of two 'structural

152 maintenance of chromosomes' (SMC) proteins, SMC2 and SMC4, and are distinguished by three unique

153 CAP (chromosome-associated polypeptide) proteins named CAPG, CAPD and CAPH (47). Uniquely,

154 C. elegans has an additional complex, condensin-IDC, which contributes exclusively to dosage

155 compensation in somatic cells (48). KLE-2, HCP-6 and CAPG-2 are condensin-II specific subunits,

156 CAPG-1, DPY-26 and DPY-28 are common to the two condensin-I complexes, whereas DPY-27 is

157 specific to condensin-IDC (Figure 2A) (46). In C. elegans, condensin-II associates with sister chromatids

158 in meiosis and mediates their compaction and resolution $(49,50)$. Because depletion of condensin-II

159 subunits results in sterility, we could not study the interaction of single condensin-II mutants with set-

160 2. Instead, we knocked-down different subunits of condensin in wildtype and set-2 mutant animals by

161 growing animals from the L1 larval stage to adulthood on condensin RNAi feeding plates, followed by

162 scoring of DAPI stained germlines by fluorescence microscopy (Figure 2B and D). We first focused on

163 kle-2 and capg-1 RNAi to knock-down condensin-II and condensin-I complexes, respectively. RT-

164 qPCR analysis showed that RNAi treatments resulted in a similar decrease in transcript levels in both

165 wildtype and set-2 mutant animals (Figure 2C), confirming that the efficiency of RNAi was the same in

166 both contexts.

167 Condensin-II RNAi resulted in partial sterility in both wildtype and mutant animals. Because

168 many of these animals also showed somatic defects that prevented egg-laying, we were unable to use

169 the number of progeny laid as a read-out of fertility in these experiments. We instead turned to visual

170 scoring of germlines, placing animals in one of two broad classes: "wildtype-like" or "abnormal"

171 (Figures 2B and D). Germlines in the wildtype-like class consisted of nuclei undergoing all stages of

172 meiotic progression as in wild type, although the total number of germ cells was reduced, consistent

173 with the severe under-proliferation observed in condensin-II mutants (46). The second class defined as

174 "abnormal" consisted of severely disorganized germlines containing fewer and larger nuclei, often 

multiple spots in enlarged nuclei, revealing that these were aneuploid nuclei (Figure S3). The abnormal germline morphology of these mutants made it difficult to clearly distinguish different region of the germline, and individual cells could not be unequivocally assigned to a specific meiotic stages. Nuclei were sometimes connected by thin chromatin bridges (Figure 2B, arrow), consistent with the known involvement of condensin-II in chromosome segregation in the germline and soma (46)(52). RNAi of the condensin-II subunit kle-2 in wildtype animals resulted in an equal number of germlines falling in the wildtype-like and abnormal class (Figure 2B). kle-2(RNAi) in set-2 mutant animals resulted in similar phenotypes, but the number of germlines showing an abnormal phenotype was significantly higher, representing $80 \%$ of all germlines in blind scoring experiments. Similar results were observed following RNAi knock-down of the other condensin-II specific subunits, $h c p-6$ and capg-

187 2, as well as $s m c-4$, common to both condensin I and II complexes. In all conditions, phenotypes were 188 consistently and significantly more severe in set-2 mutant animals than in wildtype animals (Figure 2D).

189 Knockdown of the condensin-I specific subunits capg- 1 and $d p y-28$, or the condensin-IDC subunit $d p y$ -

19027 , did not produce any apparent germline phenotype, either alone, or in the set-2 mutant background 191 (Figure 2D) (46,49,50). The effectiveness of capg-1, dpy-28 and dpy-27 RNAi was confirmed by scoring 192 the associated dumpy (Dpy) phenotype in wildtype and mutant animals (Figure S4;

193 www.wormbase.com). In summary, germline phenotypes resulting from condensin-II knock-down are

194 significantly and reproducibly more severe in the absence of set-2, and these marked defects are not 195 observed following knock-down of the other two condensin complexes in C. elegans. Importantly, 196 transcriptome profiling of set-2 mutant germlines did not show mis-regulation of condensin subunits, or 197 of other genes with obvious functions in chromosome organization, further supporting a transcription198 independent effect (Table S1). Altogether, these results strongly suggest that set-2 contributes to proper 199 meiotic chromosome organization in the C. elegans germline, a process largely dependent on condensin200 II activity.

201 Increased sterility and germline apoptosis in hcp-6(mr17);set-2 double mutants 
202 To validate the above results based on RNAi knock-down, we used $m r 17$, a hypomorphic allele of the 203 condensin-II subunit $h c p-6$ that carries a missense mutation resulting in temperature-sensitive 204 embryonic lethality (52). In their previous analysis, the authors identified it as a $\mathrm{G}$ to A transition at 205 nucleotide position 3073 within the coding region, converting glycine 1024 to glutamic acid (52). We 206 could not confirm the identity of this mutation by resequencing. Instead, we identified a glycine to 207 glutamic acid substitution at amino acid 683 within the HEAT repeat of HCP-6 (Figure 3A).

Because the germlines of $h c p-6(m r 17)$ single and double mutants are severely disorganized at $25^{\circ} \mathrm{C}$ (Figure S5 A), we were unable to visually score distinct phenotypic classes in single compared to $h c p-6(m r 17) ;$ set-2 double mutants, as we did for the RNAi experiments. However, we observed that $h c p-6(m r 17)$ mutants showed a significant reduction in the number of progeny laid at all temperatures tested $\left(15^{\circ}, 20^{\circ}, 25^{\circ} \mathrm{C}\right)$ (Figure 3B), consistent with the essential role of condensin-II in germline fertility (46). Using total brood size as a read-out of germline health, we found that set-2 single mutants laid 214 fewer eggs at all temperatures tested, as previously described (22). Brood size was further and 215 significantly reduced in $h c p-6(m r 17)$;set-2 double mutants compared to either of the single mutants at 216 all temperatures (Figure 3B). Furthermore, in all cases, the $h c p-6($ mr 17); set-2 double mutant phenotype 217 was most severe at the non-permissive temperature of $25^{\circ} \mathrm{C} . h c p-6(\mathrm{mr} 17)$; set-2 double mutants laid a 218 mean of 30 embryos per animal at $25^{\circ} \mathrm{C}$, compared to $250-300$ for wild type, showing that germline 219 function was severely impacted in these animals.

Defective germline nuclei in C. elegans hermaphrodites are eliminated by apoptosis $(53,54)$, 221 which could contribute to the reduced brood size of $h c p-6$ mutant animals. Using the dye acridine orange 222 as a marker of apoptotic cells (53), we observed as expected an increase in apoptosis in brc-1/BRCA1 223 mutants (55), while set-2 single mutants were unaffected, as previously shown (Figures 3C and D)(24). 224 The number of apoptotic corpses was increased in hcp-6 mutants, with a significant further increase in set-2;hcp-6(mr17) double mutants. The elimination of defective germline nuclei by apoptosis may 226 therefore contribute to the reduced fertility of these animals. Alternative, or in addition, a decrease in 227 the mitotic stem cell population may contribute to the observed reduction in brood size. Altogether, our 228 results are consistent with RNAi knock-down experiments and suggest that condensin-II and set-2 may 229 act in similar processes in the germline. 
231 with condensin-II in embryos. To test this possibility, L4 animals raised at the permissive temperature 232 of $15^{\circ} \mathrm{C}$ were shifted to $20^{\circ} \mathrm{C}$ and allowed to develop into adults, followed by scoring of their progeny. 233 Under these conditions, $h c p-6(m r 17)$ animals showed more than $85 \%$ embryonic lethality (Figure S5 234 B). Surprisingly, we observed that embryonic lethality was significantly reduced to $65 \%$ in set-2; hcp235 6(mr17) double mutants (Figure S5 B). Animals that hatched developed into adults showing phenotypes 236 associated with cell division defects, including uncoordinated behaviour and sterility (56), suggesting 237 that partial suppression of embryonic lethality in the absence of set-2 may be independent of an effect 238 on chromosome segregation. Altogether, these results support the hypothesis of a functional interaction 239 of set-2 with condensin-II in both the germline and embryos.

set-2 inactivation also enhances chromosome organization defects of top-2(it7) mutant germlines

241 In addition to condensin complexes, topoisomerase II is another major component of mitotic 242 chromosomes. Functional loss of topoisomerase II leads to defects in chromosome morphology and 243 abnormal mitotic chromosome segregation in all species studied (57-59), including C. elegans (60).

244 When temperature-sensitive topoisomerase II mutants are used to bypass its essential requirement in 245 mitosis, defects in chromosome condensation and segregation are also observed in meiosis (61-64). 246 TOP-2, the single C. elegans topoisomerase II, shows linear localization along the exterior of mitotic chromosomes, consistent with a structural role (60). To test whether top-2 interacts with set-2 to ensure proper chromosome condensation in the germline, we constructed set-2;top-2 double mutants using a recently described allele, top-2 (it7), that was shown to result in a temperature-sensitive chromosome segregation defect in male spermatogenesis (65).

We observed that top-2(it7) hermaphrodites that developed from animals shifted to the nonpermissive temperature $\left(24^{\circ} \mathrm{C}\right)$ at the $\mathrm{L} 1$ larval stage also had defects in germline organization. Approximately one third (36\%) of adults contained normal germline arms whose size and developmental transitions were comparable to wild type (Figure 4A). The remaining animals contained either a smaller germline, such that germline bends were premature, or a germline atrophy phenotype with only a small population of mitotic germ cells. Notably, animals that displayed germline atrophy were mostly devoid 
in top-2 (it7);set-2 double than in top-2 single mutants, accounting for $65 \%$ of all germlines scored $(\mathrm{n}=400)$ (Figure 4A). In addition, we observed a minor fraction of germlines consisting of only mitotic germ cells (tumorous phenotype, data not shown). Altogether, these results show that the combined absence of top-2 and set-2 results in a dramatic germ cell atrophy.

In wildtype oocytes, individual chromosomes appear as 6 condensed DAPI stained structures (33). Consistent with the established role of topoisomerase II in sister chromatid segregation $(59,67-$ 71), top-2(it7) mutants had a significant number of oocytes (33\%) with more than 6 DAPI-stained bodies (Figure 4C and D). A similar phenotype was observed in top-2(it7);set-2 double mutants, suggesting that this particular function of topoisomerase II is independent of SET-2. Altogether, these results are consistent with a role for SET-2 in cooperation with condensin and Topo II, two major components of chromosome architecture, to achieve proper chromatin organization of pachytene chromosomes.

\section{Loss of COMPASS targeting subunit CFP-1 results in similar chromatin organization defects as}

\section{SET-2 inactivation}

We next asked whether other subunits of COMPASS also enhance the germline defects resulting from condensin-II knockdown. As observed for set-2, inactivation of $c f p-1$ using either the deletion allele tm6369 or RNAi resulted in a strong decrease in H3K4me3 in both the germline and soma $(22,72,73$ and Figure S6 A). The number of animals with an abnormal germline phenotype following RNAi of $s m c-4$, targeting condesin-I and -II, or kle-2, targeting condensin-II only, was largely increased in $c f p-1$ mutants compared to wild type (Figure 5A). FLIM-FRET analysis of $c f p-1$ mutants expressing H2B2FP showed a drastic nanoscale decompaction of pachytene chromatin (Figure 5B), further supporting a role for COMPASS in chromosome organization.

As in other species, H3K4me3 in C. elegans is removed by the well-conserved lysine demethylase RBR-2/KDM5, and H3K4me3 levels are strongly increased in its absence at all developmental stages (74-76). $r b r-2$ has been shown to counteract the effect of COMPASS on longevity (77), but like set-2 is required to maintain germline immortality at high temperatures (75). We found that absence of RBR-2 activity in the $r b r-2(t m 1231)$ deletion allele (75) did not result in enhancement of germline defects resulting from either kle-2 or smc-4 (RNAi) (Figure 5A). qRT-PCR analysis confirmed that although the overall efficacy of RNAi varied between independent experiments (78), 
within the same experiment efficacy was comparable in wildtype, $c f p-1$ and $r b r-2$ mutants (Figure S6

287 B). Furthermore, the percentage of animals with a strong phenotype was similar in all three experiments 288 (Figure S6 C), consistent with depletion of condensin-II below a threshold level being sufficient to 289 provoke defects in chromosome organization (81). Therefore, contrary to COMPASS inactivation, 290 increasing H3K4 methylation levels in $r b r-2$ mutants has no obvious impact on germline chromatin 291 organization.

\section{Discussion}

294 Using three different experimental approaches, we show that in the C. elegans germline the COMPASS 295 H3K4 methyltransferase complex contributes to the organization of chromosome architecture. First, using quantitative FLIM-FRET we found that chromatin compaction is affected at the nucleosomal level in live animals that lack the COMPASS subunits SET-2 and CFP-1. Second, we demonstrate that defects

298 in germline nuclei compaction following RNAi knock-down of condensin-II subunits are strongly 299 enhanced in the absence of either SET-2 or CFP-1. Third, using the number of progeny as a read-out of 300 fertility, we found that set-2 inactivation exacerbates the fertility defects associated with a hypomorphic 301 allele of the condensin-II subunit $h c p-6$.

A current model proposes that condensin complexes topologically shape mitotic chromosomes through a loop extrusion process (79), with condensin-I and -II forming arrays of helical consecutive

304 loops in mitotic cells (80). However, the fact that chromosomes still maintain a certain degree of 305 structure in the absence of both condensin-I and -II suggests that additional mechanisms and factors, 306 including histone modifying complexes and the associated modifications, may also be involved $(4,81$ 307 85,87). Using the same FLIM-FRET imaging approach as implemented here, we previously showed that 308 condensin complexes contribute to the nanoscale compaction of chromatin in the C. elegans germline 309 (43). Depletion of condensin-I affected both highly and lowly compacted regions, whereas depletion of condensin-II only affected highly compacted regions (43). Here, by quantifying nucleosomes proximity

311 using FRET imaging, we observed that the absence of set-2 affects the same compacted chromatin states 312 as the condensin-I complex (43). This suggests that set-2, and to a larger extent COMPASS as a whole, 
may contribute to the formation of loops to establish the proper structural organization of meiotic

314 chromosomes.

Because complete inactivation of condensin-II resulted in chromosome segregation defects and embryonic lethality, we used RNAi to look at the effect of mild depletion of condensin-II in the absence of COMPASS subunits. In mammals, partial inactivation of condensin by a similar approach was also 318 shown to result in relatively moderate defects in chromosome structure $(47,87-90)$. Our data show that 319 mild depletion of condensins in C. elegans results in less compacted pachytene chromosomes, and that 320 the absence of COMPASS subunits set-2 or $c f p-1$ significantly aggravates this defect. We further confirmed that COMPASS and condensin-II functionally cooperate in the germline by showing that the reduced fertility of the hypomorphic allele $h c p-6(m r 17)$ is aggravated in the absence of set- 2 . By resequencing the $h c p-6(m r 17)$ allele, we identified the mutation as a substitution of glycine with glutamic acid within one of the $\alpha$-helical HEAT repeats of HCP-6. Although the role of the HEATrepeat subunits, which are unique to eukaryotic condensins, remains largely unknown (91), our results suggest that they are of functional importance. 6(mr17) mutants at the non-permissive temperature. Out data suggest that increased apoptosis in set2; hcp-6 double mutant germlines may eliminate defective germ cells, thereby improving the quality of surviving oocytes with respect to the $h c p-6$ single mutant. Consistent with this model, increased apoptosis has also been observed in the germline of condensin-I deficient worms (92). Interestingly mutations in the BRCA1 homologs brc-1 or brd-1 are also able to partially suppress the embryonic lethality of $h c p-6(\mathrm{RNAi})$ animals, possibly reflecting a role for BRC-1/BRD-1 in the formation of toxic 334 chromatin bridges when chromosome condensation is defective (93). Our results suggest that SET-2 may also be involved in a similar process in embryos.

The observation that set-2 inactivation also enhances the defects in chromatin organization of top-2 conditional mutants further supports a structural role for SET-2 in higher-order chromatin 338 organization. Topo II and condensin are both major components of the proteinaceous mitotic scaffold 339 from which radial chromatin loops radiate $(94,95)$, and Topo II contributes to proper mitotic 340 condensation and structure in various organisms (59,81,96-103). In C. elegans, TOP-2 localizes along 
341 the chromosome length in mitosis and has been proposed to constrain chromosome length by modulating 342 chromatin loops (60). As in mitosis, topoisomerase II localizes along the chromosome axes of meiosis 343 I chromosomes in yeast and mammals $(62,63,104)$, suggesting that is may play a similar structural role 344 in organizing C. elegans germline nuclei. How might COMPASS contribute to the higher order organization of chromosomes in the $C$. 346 elegans germline? Our transcriptomic analysis of set-2 depleted germ cells reveals only minor 347 transcriptional changes (Figure S1), suggestive of a structural role rather than an indirect transcriptional 348 effect. Likewise, inactivation of the SETD1-CFP1 complex in mouse oocytes results in chromosome 349 organization defects independent of transcription $(18,105)$. We also consider a direct effect of SET-2 on condensin-II unlikely, since we found no evidence of a direct physical interaction between the two proteins in extensive proteomics analysis ((30) and data not shown). Rather, the enhancement of condensin-II knock-down phenotypes by COMPASS inactivation suggests at least partially independent, cumulative, effects on chromosome organization (Figure 6). Meiotic chromosomes from yeast to mammals are organized as linear loop arrays around a proteinaceous chromosome axis (106109), and recent super-resolution microscopy studies on mouse oocytes show that H3K4me3 emanates radially in similar structures (17). In mouse spermatocytes, strong clustering of highly transcribed loci is observed and is thought to be the result of interactions occurring locally between linear loops, as well

358 as between loci on homologs (110). Based on these observations, we suggest that the presence of 359 COMPASS at transcription sites could contribute to the organization of chromatin in these clusters. This 360 could take place through the recruitment of an H3K4me3 reader, as described for the NCAPD3 subunit 361 of condensin-II (111), or through the recruitment of additional proteins (30). Finally, given the recent 362 implication of the nuclear RNAi pathway and the MORC-1 gene silencing protein in germline chromatin 363 organization $(112,113)$, additional interactions between COMPASS and these pathways may be 364 involved. Deciphering the mechanism whereby chromatin-associated factors and histone post365 translational modifications affect global regulation of chromatin architecture in meiosis will be an 366 important area of future study.

\section{Conclusions}


368 Our studies highlight a novel role for SET1/COMPASS in chromosome organization in the C. elegans

369 germline. Given the remarkable morphological similarities between chromosomes in meiotic prophase 370 and early mitotic prophase (80,114-1119), our germline findings may have implications for mitotic 371 chromosome condensation as well.

\section{Methods}

\section{$374 \quad$ Nematode maintenance and strains}

375 Unless otherwise noted, animals were propagated under standard conditions at $20^{\circ} \mathrm{C}$ or $15^{\circ} \mathrm{C}(120)$ on 376 NGM plates (Nematode Growth Medium) seeded with the Escherichia coli strains OP50 or HT115 for 377 RNAi experiments. N2 bristol was used as the wildtype control strain. Strains used were as follows: hcp-6(mr17) I (PFR656), set-2(bn129) III/qC1 (PFR510), hcp-6(mr17) I; set-2(bn129) III (PFR651), cfp-1(tm6369) IV (PFR588), brc-1(tm1145) III (DW102), rbr-2(tm1231) IV (PFR394), oxIs279[Ppie 1::GFP::H2B + unc-119(+)] II; unc-119(ed3) III (EG4601), oxIs279[Ppie-1::GFP::H2B + unc-119(+)] II; set-2(bn129) III (PFR326), oxIs279[Ppie-1::GFP::H2B + unc-119(+)] II; cfp-1(tm6369) IV (PFR667), oxSi487 [mex-5p::mCherry::H2B::tbb-2 3'UTR::gpd-2 operon::GFP::H2B::cye-1 3'UTR + unc-119(+)] II; unc-119(ed3) III (EG6787), oxSi487 [mex-5p::mCherry::H2B::tbb-2 3'UTR::gpd-2 operon::GFP::H2B::cye-1 3'UTR + unc-119(+)] II; set-2(bn129) III (PFR659), oxSi487 [mex5p::mCherry::H2B::tbb-2 3'UTR::gpd-2 operon::GFP::H2B::cye-1 3'UTR + unc-119(+)] II; cfp1(tm6369) IV (PFR666), unc-119(ed3) III; top-2(it7) II (PFR704), top-2(it7) II; set-2(bn129) III

387 (PFR705)

388 Worm Live-Imaging Preparation

389 For FRAP and FLIM-FRET acquisitions, single worms (24 hours post-L4 stage) from an 390 unsynchronized population were picked to an unseeded 1xNGM plate to wash off bacteria and were 391 subsequently transferred onto a glass slide in a drop of egg buffer $(118 \mathrm{mM} \mathrm{NaCl}, 48 \mathrm{mM} \mathrm{KCl}, 2 \mathrm{mM}$ $392 \mathrm{CaCl}_{2} * 2 \mathrm{H}_{2} \mathrm{O}, 2 \mathrm{mM} \mathrm{MgCl} 2 * 6 \mathrm{H}_{2} \mathrm{O}, 25 \mathrm{mM}$ HEPES $\mathrm{pH}$ 7.3). Worm gonads were extruded by 393 microdissection using a 23G syringe and immediately covered with a coverslip, sealed with nail varnish.

\section{FLIM-FRET Acquisition}


FLIM-FRET measurements were carried out on wt, set-2(bn129) and cfp-1(tm6369) mutant strains

396 GFP-H2B (donor alone: GFP-H2B protein) and H2B-2FPs (donor and acceptor: GFP-H2B and 397 mCherry-H2B). FLIM was performed using an inverted laser scanning multi-photon LSM780 398 microscope (Zeiss) equipped with an environmental black-walled chamber. Measurements were 399 performed at $20^{\circ} \mathrm{C}$ with a $40 \mathrm{x}$ oil immersion lens, NA 1.3 Plan-Apochromat objective, from Zeiss. Two400 photon excitation was achieved using a tunable Chameleon Ultra II (680-1,080 nm) laser (Coherent) to 401 pump a mode-locked, frequency-doubled Ti:sapphire laser that provided sub-150-fs pulses at an 80-

402 MHz repetition rate. GFP and mCherry fluorophores were used as a FRET pair. The optimal two-photon 403 excitation wavelength to excite the donor GFP was determined to be $890 \mathrm{~nm}$ (37). Laser power was 404 adjusted to give a mean photon count rate of about $7.10^{4}-10^{5}$ photons per second. Fluorescence lifetime 405 measurements were acquired over $60 \mathrm{~s}$. Detection of the emitted photons was achieved through the use 406 of an HPM-100 module (Hamamatsu R10467-40 GaAsP hybrid photomultiplier tube [PMT]). and 407 fluorescence lifetimes were calculated for all pixels in the field of view ( 256 x 256 pixels). The 408 fluorescence lifetime imaging capability was provided by time-correlated single- photon counting 409 (TCSPC) electronics (SPC-830; Becker \& Hickl). TCSPC measures the time elapsed between laser 410 pulses and the fluorescence photons. Specific regions of interest (e.g., full gonad or pachytene nuclei) 411 were selected using SPCImage software (Becker \& Hickl).

\section{FLIM-FRET Analysis}

413 FLIM measurements were analyzed as described previously (43) using SPCImage software (Becker \& 414 Hickl). Briefly, FRET results from direct interactions between donor and acceptor molecules (121) and 415 causes a decrease in the fluorescence lifetime of the donor molecules (GFP). The FRET efficiency (i.e., 416 coupling efficiency) was calculated by comparing the fluorescence lifetime values from FLIM measurements obtained for GFP donor fluorophores in the presence and absence of mCherry acceptor

418 fluorophores. The FRET percentage images were calculated such as, $E F R E T=1-(\tau D A / \tau D)$, where $\tau \mathrm{DA}$ 419 is the mean fluorescence lifetime of the donor (GFP-H2B) in the presence of the acceptor (mCherry$420 \mathrm{H} 2 \mathrm{~B}$ ) expressed in C. elegans $2 \mathrm{~B}-2 \mathrm{FPs}$, and $\tau \mathrm{D}$ is the mean fluorescence lifetime of the donor (GFP-H2B) 421 expressed in C. elegansGFP-H2B in the absence of the acceptor. In the non-FRET conditions, the mean 
422 fluorescence lifetime value of the donor was calculated from a mean of the $\tau \mathrm{D}$ by applying a mono-

423 exponential decay model to fit the fluorescence lifetime decays.

\section{Condensin RNAi knockdown}

425 Bacterial clones expressing RNA targeting condensin-I and -II subunit were from the C. elegans RNAi 426 collection (Ahringer laboratory-Gene Service Inc). Inserts from each RNAi clone were amplified by 427 PCR on isolated colonies, with a single primer in the duplicated T7 promoter (5', 428 TAATACGACTCACTATAGGG 3'), then sequenced using the primer 5'

429 GGTCGACGGTATCGATAAGC 3'. RNAi clones were cultured in LB liquid medium supplemented 430 with $50 \mu \mathrm{g} / \mathrm{ml}$ Ampicillin for $18 \mathrm{~h}$ at $37^{\circ} \mathrm{C}$, IPTG was then added ( $1 \mathrm{mM}$ final), and cultures grown an 431 additional $2 \mathrm{~h} 30$ at $37^{\circ} \mathrm{C}$. NGM plates complemented with IPTG $(1 \mathrm{mM})$ were seeded with $300 \mu$ of 432 bacterial culture. Synchronized L1 were placed on RNAi plates and grown to adulthood.

\section{RNA isolation and qRT-PCR analysis}

434 Synchronized L1 wildtype or set-2(bn129) mutant worms were grown on empty vector L4440 or kle-2 435 or capg-1 RNAi to adult staged worms at $20^{\circ} \mathrm{C}$ and harvested. Total RNA was isolated using NucleoZol 436 (Macherey Nagel, \#740404-200) and NucleoSpin (Macherey Nagel, \#40609). RNA was reverse 437 transcribed using cDNA transcriptor (Roche, \#5893151001). Quantitative PCR analysis was performed 438 on CFX Connect (Bio-rad CFX Connect) with SYBR Green RT-PCR (Roche, \#4913914001 ). Melting 439 curve analysis was performed for each primer set to ensure the specificity of the amplified product and 440 with an efficiency of 2. pmp-3 and $c d c-42$ were used as the internal controls so that the RNA level of 441 each gene of interest was normalized to the levels of $p m p-3$ and $c d c-42$. qRT-PCR were performed on 442 three biological replicas in technical duplicates. Statistical analysis was performed using an unpaired t443 test. Primers used were:

444 pmp-3: 5' GTTCCCGTGTTCATCACTCAT 3' - 5' ACACCGTCGAGAAGCTGTAGA 3' $445 c d c-42:$ 5' CTGCTGGACAGGAAGATTACG 3' - 5' CTCGGACATTCTCGAATGAAG 3' 446 kle-2: 5' GAGAAAACGGACAGCTCGTGTG 3' - 5' CGTCATATTCAGCTCCGAGGGT 3’ 447 capg-1: 5’ TCGAATTGGCCAGTAGATGC 3' - 5' ACTGCAACAAGTCGGCATTC 3’

\section{Hoechst staining on dissected germlines}


Germlines from condensin RNAi knock-down animals were dissected on L-polylysine coated slides in

450 a drop of dissection buffer (0.4X M9 and Levamisole 20mM). After removing dissection buffer using a 451 drawn capillary, gonads were fixed in $11 \mu \mathrm{l}$ of $3 \%$ paraformaldehyde for $5 \mathrm{~min}$. Slides were washed in 452 1X PBS 0.2\% Tween 20 plus $5 \mu \mathrm{g} / \mathrm{ml}$ Hoechst 33342 (Sigma Aldrich, \#861405) for $10 \mathrm{~min}$, then twice 453 in 1 X PBS $0.2 \%$ Tween 20 for $10 \mathrm{~min}$, and mounted in mounting media (1X PBS, 4\% n-Propyl-Gallate, $45490 \%$ DE Glycerol). Z-stack images ( $0.25 \mu \mathrm{m}$ slices) of germlines were acquired using a Zeiss LSM710 455 inverted confocal microscope with a 40X oil immersion objective.

\section{DAPI staining on whole animals}

457 For scoring topo II mutant germlines, adult animals were stained as previously described (122) with 458 minor modifications. Briefly, animals were collected and washed once in $1 \mathrm{X} \mathrm{M9}$, fixed 15 min in $-20^{\circ} \mathrm{C}$ 459 methanol, and washed twice in $1 \mathrm{X}$ PBS with $0.1 \%$ Tween 20 (Sigma Aldrich, \#P1379). $25 \mu 1$ 460 Fluoroshield plus DAPI (Sigma Aldrich, \#F6057) was added directly to 50 $\mu$ l of worm pellet, followed 461 by mounting for fluorescent microscopy. Observation were made on an AxioImager A2 (Zeiss) with 462 Plan Apochromat 63X/1.4 oil DIC or EC plan Neofluar 20X/0.5 objectives.

\section{Scoring of germline phenotypes}

464 Blind scoring was carried out using AxioImager A2 (Zeiss) with EC plan Neofluar 20X/0.5 objectives. 465 For condensin RNAi knock-down, the "strong phenotype" was defined as germlines containing fewer, 466 abnormally sized and unevenly distributed nuclei, as well as macro nuclei with strong DAPI signal, as 467 previously described (46). "weak phenotype" includes wildtype-like germlines that sometimes contained 468 a few macro nuclei with strong DAPI signal. For each experiment, at least 200 germlines were scored 469 for each genotype, and at least 3 independent biological replicates were performed. For experiments 470 with the top-2(it7) allele, wildtype, set-2(bn129) and top-2(it7) single, and set-2(bn129);top-2(it7) 471 double mutants were synchronized at the $\mathrm{L} 1$ stage at $15^{\circ} \mathrm{C}$, then transfer on plates seeded with OP50 at $47224^{\circ} \mathrm{C}$ and allowed to develop to adulthood. Adults were recovered in 1X M9 and DAPI stained as 473 described in DAPI staining on whole animals. Germlines were place in phenotypic categories based on 474 (123). Data were collected from 3 independent experiments.

\section{Sequencing and mapping of the $h c p-6(\mathrm{mr} 17)$ mutation}


476 Genomic DNA from wildtype animals (N2) and from strains bearing the hcp-6(mr17) mutation was

477 amplified using a high-fidelity polymerase (Phusion ${ }^{\circledR}$, NEB \#M0530S) and the following primer pairs:

478 5' ATAGTCAACCTCGATTGCTGGCTG 3' - 5' GAGGGCGAATAAGTCTTCCGTAAG 3'

479 5’ GGAGTTTCTGCTGCCAGTAGTTAT 3' - 5’' TGTGGATAAACGTGGCGATA 3'

480 5' GATCGTtGGAGCGATTtACGGATC 3' - 5’ TGTGGATAAACGTGGCGATA 3'

481 5' GATCGTTGGAGCGATTTACGGATC 3' - 5' CTTTCTGGCATGTTCAGTGACGTC 3’

482 5' GAAATCCCGAAGCAAGAGAG 3' - 5' GTCCATGTGAGATCCGATGAGT 3'

483 5' GAAATCCCGAAGCAAGAGAG 3' - 5' CTTTCTGGCATGTTCAGTGACGTC 3’

484 5' TGGCTTCACACCTTGATCTCGATG 3' - 5' TCTTCATCGTGACCAACTCCAACC 3'

485 5’ TCTCAACGTGGCATCTGAAG 3’ - 5’ GCGTGTCGACGAACAATAAC 3’

486 5' GTTCGGAATGACGCAAAACT 3' - 5' CACAGTTTTCTCCGCATCAACATG 3’'

487 5' CACTGAAATGCGCCTTAATCCTCC 3' - 5' TGATATGGGAGGAGCTGTGAAGGA 3’

488 For each DNA fragment amplified by PCR, both forward and reverse primers were used in the

489 sequencing reactions. The presence of the $m r 17$ mutation was confirmed in 7 independent sequencing

490 reactions from mutant animals. 2 independent reactions from wildtype animals were used as reference.

\section{Brood size and embryonic lethality assays}

492 To score fertility and embryonic lethality, 10 to 11 individual $\mathrm{L} 4$ hermaphrodites grown at $15^{\circ} \mathrm{C}$ were 493 picked and transferred to individual plates at either $15^{\circ} \mathrm{C}, 20^{\circ} \mathrm{C}$ or $25^{\circ} \mathrm{C}$. Animals were transferred on 494 new plates until they stopped laying eggs, and the number of eggs on individual plates scored each day. 495 After 24h, unhatched eggs and live progeny were scored. Embryonic lethality represents the number of 496 unhatched eggs, divided by the total number of total eggs laid. Experiments were repeated 3 times each.

497 Visualization of apoptotic cells in the germline

498 Acridine Orange (Sigma Aldrich \#A9231) was used to visualized apoptotic cells in the germline of live 499 animals as previously described (124). Briefly, L4 hermaphrodites grown at permissive temperature $500\left(15^{\circ} \mathrm{C}\right)$, were placed on NGM plates at the restrictive temperature of $20^{\circ} \mathrm{C}$ during $18 \mathrm{~h} .1 \mathrm{ml}$ of Acridine 501 Orange diluted at a final concentration of $50 \mu \mathrm{g} / \mathrm{ml}$ in M9 buffer was added to the plates and incubated 502 for $2 \mathrm{~h}$ in the dark. Stained animals were transferred to a fresh NGM plates seeded with OP50 and 503 incubated for $2 \mathrm{~h}$ in the dark in order to remove stained bacteria in the intestine. Animals were placed on 
4\% agar pad in a drop of 10mM levamisole (Sigma Aldrich \#L9756) diluted in M9 buffer, a coverslip

505 was placed on top and sealed with nailed polish. Z-stack images of the posterior gonad were acquired 506 using a Zeiss LSM710 inverted confocal microscope with 40X oil Immersion objective. Z-stack of 507 germlines were acquire every $0.5 \mu \mathrm{m}$, images correspond to a projection using Max intensity method 508 using Fiji (125). At least 20 gonads were imaged for each genotype.

509

510 Declarations

511 Ethics approval and consent to participate: not applicable

512 Consent for publication: not applicable

513 Availability of data and materials: All data generated or analysed during this study are

514 included in this published article [and its supplementary information files]. Gene expression

515 data have been deposited at GEO (awaiting processing)

\section{Competing interests}

517 The authors declare that they have no competing interests

\section{$518 \quad$ Funding}

519 This work was supported by the ANR ( $N^{\circ}$ 15-CE12-0018-01), the CNRS, and the Fondation ARC

\section{Authors' contributions}

521 FP and MH designed genetic experiments; MH performed and analyzed genetic assays; DL carried out 522 FRAP and FLIM-FRET experiments; AB made genetic constructs and helped with FLIM-FRET 523 acquisition and experimental design; VR helped with experimental design and helped carry out qRT524 PCR analysis; MH and LG carried out immunofluorescence analysis; FP and DL wrote the paper; MH, 525 FP and DL prepared figures, and all authors helped with editing; all authors read and approved the final 526 manuscript

\section{Acknowledgements}

528 We thank "Montpellier Ressources Imagerie" (MRI), CGC, which is funded by NIH Office of Research Infrastructure Programs (P40 OD010440) for strains, and the Mitani lab for deletion strains. We 
gratefully acknowledge support from the PSMN (Pôle Scientifique de Modélisation Numérique) of the

531 ENS de Lyon for computing resources.

\section{References}

535 1. Rowley MJ, Corces VG. Organizational principles of 3D genome architecture. Nat Rev Genet. 2018 Dec;19(12):789-800.

537 2. Antonin W, Neumann H. Chromosome condensation and decondensation during mitosis. Curr Opin Cell Biol. 2016;40:15-22.

3. Beck DB, Oda H, Shen SS, Reinberg D. PR-Set7 and H4K20me1: at the crossroads of genome

4. Zhiteneva A, Bonfiglio JJ, Makarov A, Colby T, Vagnarelli P, Schirmer EC, et al. Mitotic postintegrity, cell cycle, chromosome condensation, and transcription. Genes Dev. 2012 Feb 15;26(4):325-37.

5. Hayashi K, Yoshida K, Matsui Y. A histone H3 methyltransferase controls epigenetic events

6. Borde V, Robine N, Lin W, Bonfils S, Geli V, Nicolas A. Histone H3 lysine 4 trimethylation

7. Buard J, Barthès P, Grey C, de Massy B. Distinct histone modifications define initiation and translational modifications of histones promote chromatin compaction in vitro. Open Biol. 2017;7(9).

8. Baudat F, Buard J, Grey C, Fledel-Alon A, Ober C, Przeworski M, et al. PRDM9 is a major determinant of meiotic recombination hotspots in humans and mice. Science. 2010 Feb 12;327(5967):836-40.

9. Eissenberg JC, Shilatifard A. Histone H3 lysine 4 (H3K4) methylation in development and

10. Dehe PM, Dichtl B, Schaft D, Roguev A, Pamblanco M, Lebrun R, et al. Protein interactions within the Set 1 complex and their roles in the regulation of histone 3 lysine 4 methylation. J Biol Chem. 2006 Nov 17;281(46):35404-12.

11. Howe FS, Fischl H, Murray SC, Mellor J. Is H3K4me3 instructive for transcription activation?

12. Vermeulen M, Mulder KW, Denissov S, Pijnappel WW, van Schaik FM, Varier RA, et al. Oct 5;131(1):58-69. 
14. Taverna SD, Ilin S, Rogers RS, Tanny JC, Lavender H, Li H, et al. Yng1 PHD finger binding to $\mathrm{H} 3$ trimethylated at $\mathrm{K} 4$ promotes $\mathrm{NuA} 3 \mathrm{HAT}$ activity at $\mathrm{K} 14$ of $\mathrm{H} 3$ and transcription at a subset of targeted ORFs. Mol Cell. 2006 Dec 8;24(5):785-96.

15. Yun M, Wu J, Workman JL, Li B. Readers of histone modifications. Cell Res. 2011 Apr;21(4):564-78.

16. Lam K-WG, Brick K, Cheng G, Pratto F, Camerini-Otero RD. Cell-type-specific genomics reveals histone modification dynamics in mammalian meiosis. Nat Commun. 2019 Dec;10(1):3821.

17. Prakash K, Fournier D, Redl S, Best G, Borsos M, Tiwari VK, et al. Superresolution imaging reveals structurally distinct periodic patterns of chromatin along pachytene chromosomes. Proc Natl Acad Sci U S A. 2015 Nov 24;112(47):14635-40.

18. Sha Q-Q, Dai X-X, Jiang J-C, Yu C, Jiang Y, Liu J, et al. CFP1 coordinates histone H3 lysine-4 trimethylation and meiotic cell cycle progression in mouse oocytes. Nat Commun. 2018 28;9(1):3477.

19. Mikheyeva IV, Grady PJR, Tamburini FB, Lorenz DR, Cam HP. Multifaceted genome control by Set1 Dependent and Independent of H3K4 methylation and the Set1C/COMPASS complex. PLoS Genet. 2014 Oct;10(10):e1004740.

20. Mizuguchi T, Barrowman J, Grewal SIS. Chromosome domain architecture and dynamic organization of the fission yeast genome. FEBS Lett. 2015 Oct 7;589(20PartA):2975-86.

21. Lorenz DR, Mikheyeva IV, Johansen P, Meyer L, Berg A, Grewal SIS, et al. CENP-B cooperates with Set1 in bidirectional transcriptional silencing and genome organization of retrotransposons. Mol Cell Biol. 2012 Oct;32(20):4215-25.

22. Xiao Y, Bedet C, Robert VJ, Simonet T, Dunkelbarger S, Rakotomalala C, et al. Caenorhabditis elegans chromatin-associated proteins SET-2 and ASH-2 are differentially required for histone H3 Lys 4 methylation in embryos and adult germ cells. Proc Natl Acad Sci U A. 2011 May 17;108(20):8305-10.

23. Li T, Kelly WG. A role for Set1/MLL-related components in epigenetic regulation of the Caenorhabditis elegans germ line. PLoS Genet. 2011 Mar;7(3):e1001349.

24. Herbette M, Mercier MG, Michal F, Cluet D, Burny C, Yvert G, et al. The C. elegans SET2/SET1 histone H3 Lys4 (H3K4) methyltransferase preserves genome stability in the germline. DNA Repair. 2017 Sep;57:139-50.

25. Robert VJ, Mercier MG, Bedet C, Janczarski S, Merlet J, Garvis S, et al. The SET-2/SET1 histone H3K4 methyltransferase maintains pluripotency in the Caenorhabditis elegans germline. Cell Rep. 2014 Oct 23;9(2):443-50.

26. Clouaire T, Webb S, Skene P, Illingworth R, Kerr A, Andrews R, et al. Cfp1 integrates both CpG content and gene activity for accurate H3K4me3 deposition in embryonic stem cells. Genes Dev. 2012 Aug 1;26(15):1714-28.

27. Clouaire T, Webb S, Bird A. Cfp1 is required for gene expression-dependent H3K4 trimethylation and H3K9 acetylation in embryonic stem cells. Genome Biol. 2014 Sep 4;15(9):451. 
29. Weiner A, Chen HV, Liu CL, Rahat A, Klien A, Soares L, et al. Systematic dissection of roles for chromatin regulators in a yeast stress response. PLoS Biol. 2012 Jul;10(7):e1001369.

30. Beurton F, Stempor P, Caron M, Appert A, Dong Y, Chen RA-J, et al. Physical and functional interaction between SET1/COMPASS complex component CFP-1 and a Sin3S HDAC complex in C. elegans. Nucleic Acids Res. 2019 Dec 2;47(21):11164-80.

31. Hirano T. Condensins: universal organizers of chromosomes with diverse functions. Genes Dev. 2012 Aug 1;26(15):1659-78.

32. Piskadlo E, Oliveira RA. Novel insights into mitotic chromosome condensation. F1000Research. 2016;5.

33. Kimble J, Crittenden SL. Germline proliferation and its control. WormBook Online Rev C

34. Fisher K, Southall SM, Wilson JR, Poulin GB. Methylation and demethylation activities of a C.

35. Engert CG, Droste R, van Oudenaarden A, Horvitz HR. A Caenorhabditis elegans protein with a PRDM9-like SET domain localizes to chromatin-associated foci and promotes spermatocyte gene expression, sperm production and fertility. PLoS Genet. 2018;14(4):e1007295.

36. Adriaens C, Serebryannyy LA, Feric M, Schibler A, Meaburn KJ, Kubben N, et al. Blank spots on the map: some current questions on nuclear organization and genome architecture. Histochem Cell Biol. 2018 Dec;150(6):579-92.

37. Llères D, James J, Swift S, Norman DG, Lamond AI. Quantitative analysis of chromatin compaction in living cells using FLIM-FRET. J Cell Biol. 2009 Nov 16;187(4):481-96.

38. Lou J, Scipioni L, Wright BK, Bartolec TK, Zhang J, Masamsetti VP, et al. Phasor histone FLIMFRET microscopy quantifies spatiotemporal rearrangement of chromatin architecture during the DNA damage response. Proc Natl Acad Sci. 2019 Apr 9;116(15):7323-32.

39. Sobecki M, Mrouj K, Camasses A, Parisis N, Nicolas E, Llères D, et al. The cell proliferation

40. Baarlink C, Plessner M, Sherrard A, Morita K, Misu S, Virant D, et al. A transient pool of nuclear F-actin at mitotic exit controls chromatin organization. Nat Cell Biol. 2017 Dec;19(12):138999.

41. Wang Y, Sherrard A, Zhao B, Melak M, Trautwein J, Kleinschnitz E-M, et al. GPCR-induced calcium transients trigger nuclear actin assembly for chromatin dynamics. Nat Commun. 2019 Dec;10(1):5271.

42. Llères D, Swift S, Lamond AI. Detecting Protein-Protein Interactions In Vivo with FRET using Multiphoton Fluorescence Lifetime Imaging Microscopy (FLIM). Curr Protoc Cytom [Internet]. 2007 Oct [cited 2020 Feb 28];42(1). Available from: https://onlinelibrary.wiley.com/doi/abs/10.1002/0471142956.cy1210s42

43. Llères D, Bailly AP, Perrin A, Norman DG, Xirodimas DP, Feil R. Quantitative FLIM-FRET Microscopy to Monitor Nanoscale Chromatin Compaction In Vivo Reveals Structural Roles of Condensin Complexes. Cell Rep. 2017 Feb 14;18(7):1791-803. 
45. Beurton F. Physical and functional interaction between the SET1 complex component CFP1/CXXC and the Sin-3S/HDAC complex at promoter regions. Nucleic Acids Res. 2019 Dec 2;47(21):11164-80.

46. Csankovszki G, Collette K, Spahl K, Carey J, Snyder M, Petty E, et al. Three distinct condensin complexes control C. elegans chromosome dynamics. Curr Biol CB. 2009 Jan 13;19(1):9-19.

47. Ono T, Fang Y, Spector DL, Hirano T. Spatial and temporal regulation of Condensins I and II in mitotic chromosome assembly in human cells. Mol Biol Cell. 2004 Jul;15(7):3296-308.

48. Albritton SE, Ercan S. Caenorhabditis elegans Dosage Compensation: Insights into CondensinMediated Gene Regulation. Trends Genet TIG. 2018;34(1):41-53.

49. Chan RC, Severson AF, Meyer BJ. Condensin restructures chromosomes in preparation for meiotic divisions. J Cell Biol. 2004 Nov 22;167(4):613-25.

50. Mets DG, Meyer BJ. Condensins regulate meiotic DNA break distribution, thus crossover frequency, by controlling chromosome structure. Cell. 2009 Oct 2;139(1):73-86.

51. Gonzalez-Serricchio AS, Sternberg PW. Visualization of C. elegans transgenic arrays by GFP. BMC Genet. 2006 Jun 7;7:36.

52. Stear JH, Roth MB. Characterization of HCP-6, a C. elegans protein required to prevent chromosome twisting and merotelic attachment. Genes Dev. 2002 Jun 15;16(12):1498-508.

53. Gumienny TL, Lambie E, Hartwieg E, Horvitz HR, Hengartner MO. Genetic control of programmed cell death in the Caenorhabditis elegans hermaphrodite germline. Development. 1999 Feb;126(5):1011-22.

54. Gartner A, Boag PR, Blackwell TK. Germline survival and apoptosis. WormBook Online Rev C Elegans Biol. 2008 Sep 4;1-20.

55. Boulton SJ, Martin JS, Polanowska J, Hill DE, Gartner A, Vidal M. BRCA1/BARD1 orthologs required for DNA repair in Caenorhabditis elegans. Curr Biol. 2004 Jan 6;14(1):33-9.

56. O'Connell KF, Leys CM, White JG. A genetic screen for temperature-sensitive cell-division mutants of Caenorhabditis elegans. Genetics. 1998 Jul;149(3):1303-21.

57. Adachi Y, Luke M, Laemmli UK. Chromosome assembly in vitro: topoisomerase II is required for condensation. Cell. 1991 Jan 11;64(1):137-48.

58. Hirano T, Mitchison TJ. Topoisomerase II does not play a scaffolding role in the organization of mitotic chromosomes assembled in Xenopus egg extracts. J Cell Biol. 1993 Feb;120(3):601-12.

59. Uemura T, Ohkura H, Adachi Y, Morino K, Shiozaki K, Yanagida M. DNA topoisomerase II is required for condensation and separation of mitotic chromosomes in S. pombe. Cell. 1987 Sep;50(6):917-25.

60. Ladouceur A-M, Ranjan R, Smith L, Fadero T, Heppert J, Goldstein B, et al. CENP-A and topoisomerase-II antagonistically affect chromosome length. J Cell Biol. 2017 04;216(9):264555.

61. Marchetti F, Bishop JB, Lowe X, Generoso WM, Hozier J, Wyrobek AJ. Etoposide induces heritable chromosomal aberrations and aneuploidy during male meiosis in the mouse. Proc Natl Acad Sci. 2001 Mar 27;98(7):3952-7. 
62. Li X-M, Yu C, Wang Z-W, Zhang Y-L, Liu X-M, Zhou D, et al. DNA Topoisomerase II Is

64. Hughes SE, Hawley RS. Topoisomerase II is required for the proper separation of heterochromatic regions during Drosophila melanogaster female meiosis. PLoS Genet. 2014 Oct;10(10):e1004650.

65. Jaramillo-Lambert A, Fabritius AS, Hansen TJ, Smith HE, Golden A. The Identification of a Novel Mutant Allele of topoisomerase II in Caenorhabditis elegans Reveals a Unique Role in Chromosome Segregation During Spermatogenesis. Genetics. 2016 Dec;204(4):1407-22.

66. Rog O, Dernburg AF. Chromosome pairing and synapsis during Caenorhabditis elegans meiosis. Curr Opin Cell Biol. 2013 Jun;25(3):349-56.

67. Liang Z, Zickler D, Prentiss M, Chang FS, Witz G, Maeshima K, et al. Chromosomes Progress to Metaphase in Multiple Discrete Steps via Global Compaction/Expansion Cycles. Cell. 2015 May 21;161(5):1124-37.

68. Nagasaka K, Hossain MJ, Roberti MJ, Ellenberg J, Hirota T. Sister chromatid resolution is an intrinsic part of chromosome organization in prophase. Nat Cell Biol. 2016;18(6):692-9.

69. Oliveira RA, Hamilton RS, Pauli A, Davis I, Nasmyth K. Cohesin cleavage and Cdk inhibition trigger formation of daughter nuclei. Nat Cell Biol. 2010 Feb;12(2):185-92.

70. DiNardo S, Voelkel K, Sternglanz R. DNA topoisomerase II mutant of Saccharomyces cerevisiae: topoisomerase II is required for segregation of daughter molecules at the termination of DNA replication. Proc Natl Acad Sci. 1984 May 1;81(9):2616-20.

71. Sundin O, Varshavsky A. Terminal stages of SV40 DNA replication proceed via multiply intertwined catenated dimers. Cell. 1980 Aug;21(1):103-14.

72. Chen RA-J, Stempor P, Down TA, Zeiser E, Feuer SK, Ahringer J. Extreme HOT regions are CpG-dense promoters in C. elegans and humans. Genome Res. 2014 Jul;24(7):1138-46.

73. Simonet T, Dulermo R, Schott S, Palladino F. Antagonistic functions of SET-2/SET1 and HPL/HP1 proteins in C. elegans development. Dev Biol. 2007 Dec 1;312(1):367-83.

74. Christensen J, Agger K, Cloos PA, Pasini D, Rose S, Sennels L, et al. RBP2 belongs to a family of demethylases, specific for tri-and dimethylated lysine 4 on histone 3. Cell. 2007 Mar $23 ; 128(6): 1063-76$.

75. Alvares SM, Mayberry GA, Joyner EY, Lakowski B, Ahmed S. H3K4 demethylase activities repress proliferative and postmitotic aging. Aging Cell. 2014 Apr;13(2):245-53.

76. Lussi YC, Mariani L, Friis C, Peltonen J, Myers TR, Krag C, et al. Impaired removal of H3K4 methylation affects cell fate determination and gene transcription. Dev Camb Engl. 2016 15;143(20):3751-62. 
77. Greer EL, Maures TJ, Hauswirth AG, Green EM, Leeman DS, Maro GS, et al. Members of the $\mathrm{H} 3 \mathrm{~K} 4$ trimethylation complex regulate lifespan in a germline-dependent manner in $\mathrm{C}$. elegans. Nature. 2010 Jul;466(7304):383-7.

78. Wang J, Barr MM. RNA Interference in Caenorhabditis elegans. In: Methods in Enzymology [Internet]. Elsevier; 2005 [cited 2020 Feb 11]. p. 36-55. Available from: https://linkinghub.elsevier.com/retrieve/pii/S0076687904920034

79. Ganji M, Shaltiel IA, Bisht S, Kim E, Kalichava A, Haering CH, et al. Real-time imaging of

80. Gibcus JH, Samejima K, Goloborodko A, Samejima I, Naumova N, Nuebler J, et al. A pathway

81. Samejima K, Samejima I, Vagnarelli P, Ogawa H, Vargiu G, Kelly DA, et al. Mitotic

82. Wilkins BJ, Rall NA, Ostwal Y, Kruitwagen T, Hiragami-Hamada K, Winkler M, et al. A Cascade of Histone Modifications Induces Chromatin Condensation in Mitosis. Science. 2014 Jan 3;343(6166):77-80.

83. Georgatos SD, Markaki Y, Christogianni A, Politou AS. Chromatin remodeling during mitosis: a structure-based code? Front Biosci Landmark Ed. 2009 Jan 1;14:2017-27.

84. Kruitwagen T, Denoth-Lippuner A, Wilkins BJ, Neumann H, Barral Y. Axial contraction and short-range compaction of chromatin synergistically promote mitotic chromosome condensation. eLife. 2015 Nov 28;4:e1039.

85. Markaki Y, Christogianni A, Politou AS, Georgatos SD. Phosphorylation of histone H3 at Thr3 is part of a combinatorial pattern that marks and configures mitotic chromatin. J Cell Sci. 2009 Aug 15;122(Pt 16):2809-19.

86. Batty P, Gerlich DW. Mitotic Chromosome Mechanics: How Cells Segregate Their Genome. Trends Cell Biol. 2019 Sep;29(9):717-26.

87. Vagnarelli P, Hudson DF, Ribeiro SA, Trinkle-Mulcahy L, Spence JM, Lai F, et al. Condensin and Repo-Man-PP1 co-operate in the regulation of chromosome architecture during mitosis. Nat Cell Biol. 2006 Oct;8(10):1133-42.

88. Hudson DF, Vagnarelli P, Gassmann R, Earnshaw WC. Condensin Is Required for Nonhistone Protein Assembly and Structural Integrity of Vertebrate Mitotic Chromosomes. Dev Cell. 2003 Aug;5(2):323-36.

89. Ono T, Losada A, Hirano M, Myers MP, Neuwald AF, Hirano T. Differential contributions of condensin I and condensin II to mitotic chromosome architecture in vertebrate cells. Cell. 2003 Oct 3;115(1):109-21.

90. Samoshkin A, Arnaoutov A, Jansen LET, Ouspenski I, Dye L, Karpova T, et al. Human condensin function is essential for centromeric chromatin assembly and proper sister kinetochore orientation. PloS One. 2009 Aug 28;4(8):e6831.

91. Yoshimura SH, Hirano T. HEAT repeats - versatile arrays of amphiphilic helices working in crowded environments? J Cell Sci. 2016 01;129(21):3963-70. 
92. Hernandez MR, Davis MB, Jiang J, Brouhard EA, Severson AF, Csankovszki G. Condensin I protects meiotic cohesin from WAPL-1 mediated removal. PLoS Genet. 2018;14(5):e1007382.

93. Hong Y, Sonneville R, Agostinho A, Meier B, Wang B, Blow JJ, et al. The SMC-5/6 Complex and the HIM-6 (BLM) Helicase Synergistically Promote Meiotic Recombination Intermediate Processing and Chromosome Maturation during Caenorhabditis elegans Meiosis. PLoS Genet. 2016 Mar;12(3):e1005872.

94. Belmont AS. Mitotic chromosome scaffold structure: New approaches to an old controversy. Proc Natl Acad Sci. 2002 Dec 10;99(25):15855-7.

95. Gasser SM, Laroche T, Falquet J, Boy de la Tour E, Laemmli UK. Metaphase chromosome structure. J Mol Biol. 1986 Apr;188(4):613-29.

96. Anderson H, Roberge M. Topoisomerase II inhibitors affect entry into mitosis and chromosome condensation in BHK cells. Cell Growth Differ Mol Biol J Am Assoc Cancer Res. 1996 Jan;7(1):83-90.

97. Cuvier O, Hirano T. A role of topoisomerase II in linking DNA replication to chromosome condensation. J Cell Biol. 2003 Mar 3;160(5):645-55.

98. Gonzalez RE, Lim C-U, Cole K, Bianchini CH, Schools GP, Davis BE, et al. Effects of conditional depletion of topoisomerase II on cell cycle progression in mammalian cells. Cell Cycle Georget Tex. 2011 Oct 15;10(20):3505-14.

99. Petrova B, Dehler S, Kruitwagen T, Hériché J-K, Miura K, Haering CH. Quantitative analysis of chromosome condensation in fission yeast. Mol Cell Biol. 2013 Mar;33(5):984-98.

100. Sakaguchi A, Kikuchi A. Functional compatibility between isoform alpha and beta of type II DNA topoisomerase. J Cell Sci. 2004 Mar 1;117(Pt 7):1047-54.

101. Shintomi K, Takahashi TS, Hirano T. Reconstitution of mitotic chromatids with a minimum set of purified factors. Nat Cell Biol. 2015 Aug;17(8):1014-23.

102. Buchenau P, Saumweber H, Arndt-Jovin DJ. Consequences of topoisomerase II inhibition in early embryogenesis of Drosophila revealed by in vivo confocal laser scanning microscopy. J Cell Sci. 1993 Apr;104 ( Pt 4):1175-85.

103. Vas ACJ, Andrews CA, Kirkland Matesky K, Clarke DJ. In Vivo Analysis of Chromosome Condensation in Saccharomyces cerevisiae. Bloom K, editor. Mol Biol Cell. 2007 Feb;18(2):557-68.

104. Klein F. Localization of RAP1 and topoisomerase II in nuclei and meiotic chromosomes of yeast. J Cell Biol. 1992 Jun 1;117(5):935-48.

105. Yu C, Fan X, Sha Q-Q, Wang H-H, Li B-T, Dai X-X, et al. CFP1 Regulates Histone H3K4 Trimethylation and Developmental Potential in Mouse Oocytes. Cell Rep. 2017 Aug;20(5):1161-72.

106. Blat Y, Protacio RU, Hunter N, Kleckner N. Physical and functional interactions among basic chromosome organizational features govern early steps of meiotic chiasma formation. Cell. 2002 Dec 13;111(6):791-802.

107. McNicoll F, Stevense M, Jessberger R. Cohesin in Gametogenesis. In: Current Topics in Developmental Biology [Internet]. Elsevier; 2013 [cited 2019 Oct 9]. p. 1-34. Available from: https://linkinghub.elsevier.com/retrieve/pii/B9780124160248000015 
108. Panizza S, Mendoza MA, Berlinger M, Huang L, Nicolas A, Shirahige K, et al. Spo11-accessory proteins link double-strand break sites to the chromosome axis in early meiotic recombination. Cell. 2011 Aug 5;146(3):372-83.

813

814

815

816

817

818

819

820

821

822

823

824

825

826

827

828

829

830

831

832

833

834

835

836

837

838

839

840

841

842

843

844

845

846

847

109. West AM, Rosenberg SC, Ur SN, Lehmer MK, Ye Q, Hagemann G, et al. A conserved filamentous assembly underlies the structure of the meiotic chromosome axis. eLife. 2019 Jan $18 ; 8$.

110. Patel L, Kang R, Rosenberg SC, Qiu Y, Raviram R, Chee S, et al. Dynamic reorganization of the genome shapes the recombination landscape in meiotic prophase. Nat Struct Mol Biol. 2019 Mar;26(3):164-74.

111. Yuen KC, Slaughter BD, Gerton JL. Condensin II is anchored by TFIIIC and H3K4me3 in the mammalian genome and supports the expression of active dense gene clusters. Sci Adv. 2017;3(6):e1700191.

112. Kim H, Yen L, Wongpalee SP, Kirshner JA, Mehta N, Xue Y, et al. The Gene-Silencing Protein MORC-1 Topologically Entraps DNA and Forms Multimeric Assemblies to Cause DNA Compaction. Mol Cell. 2019 22;75(4):700-710.e6.

113. Weiser NE, Yang DX, Feng S, Kalinava N, Brown KC, Khanikar J, et al. MORC-1 Integrates Nuclear RNAi and Transgenerational Chromatin Architecture to Promote Germline Immortality. Dev Cell. 2017 22;41(4):408-423.e7.

114. Alavattam KG, Maezawa S, Sakashita A, Khoury H, Barski A, Kaplan N, et al. Attenuated chromatin compartmentalization in meiosis and its maturation in sperm development. Nat Struct Mol Biol. 2019;26(3):175-84.

115. Jabbari K, Wirtz J, Rauscher M, Wiehe T. A common genomic code for chromatin architecture and recombination landscape. PloS One. 2019;14(3):e0213278.

116. Schalbetter SA, Fudenberg G, Baxter J, Pollard KS, Neale MJ. Principles of meiotic chromosome assembly revealed in S. cerevisiae. Nat Commun. 2019 22;10(1):4795.

117. Wang Y, Wang H, Zhang Y, Du Z, Si W, Fan S, et al. Reprogramming of Meiotic Chromatin Architecture during Spermatogenesis. Mol Cell. 2019 Feb;73(3):547-561.e6.

118. MacGregor IA, Adams IR, Gilbert N. Large-scale chromatin organisation in interphase, mitosis and meiosis. Biochem J. 2019 Aug 15;476(15):2141-56.

119. Naumova N, Imakaev M, Fudenberg G, Zhan Y, Lajoie BR, Mirny LA, et al. Organization of the mitotic chromosome. Science. 2013 Nov 22;342(6161):948-53.

120. Brenner S. The Genetics of Caenorhabditis elegans. Genetics. 1974;77(1):71-94.

121. T., Förster. Experimental and theoretical investigation of the intermolecular transfer of electronic excitation energy. Z Naturforsch A. 1949;4:321-7.

122. Kadyk LC, Kimble J. Genetic regulation of entry into meiosis in Caenorhabditis elegans. Dev Camb Engl. 1998 May;125(10):1803-13.

123. Heestand B, Simon M, Frenk S, Titov D, Ahmed S. Transgenerational Sterility of Piwi Mutants Represents a Dynamic Form of Adult Reproductive Diapause. Cell Rep. 2018 03;23(1):156-71. 
124. Papaluca A, Ramotar D. A novel approach using C. elegans DNA damage-induced apoptosis to characterize the dynamics of uptake transporters for therapeutic drug discoveries. Sci Rep. 2016 $27 ; 6: 36026$.

125. Schindelin J, Arganda-Carreras I, Frise E, Kaynig V, Longair M, Pietzsch T, et al. Fiji: an opensource platform for biological-image analysis. Nat Methods. 2012 Jun 28;9(7):676-82.

126. Afgan E, Baker D, Batut B, van den Beek M, Bouvier D, Cech M, et al. The Galaxy platform for accessible, reproducible and collaborative biomedical analyses: 2018 update. Nucleic Acids Res. 2018 02;46(W1):W537-44.

127. Love MI, Huber W, Anders S. Moderated estimation of fold change and dispersion for RNAseq data with DESeq2. Genome Biol. 2014;15(12):550.

128. Zhuang JJ, Banse SA, Hunter CP. The nuclear argonaute NRDE-3 contributes to transitive RNAi in Caenorhabditis elegans. Genetics. 2013 May;194(1):117-31.

\section{Figure 1. set-2 inactivation influences nanoscale chromatin compaction in the germline. (A)}

Fluorescence intensities of GFP-H2B (green) and mCherry-H2B (red) from pachytene-stage germ cells expressing H2B-2FPs. FLIM (right) images of H2B-2FPs pachytene-stage cells. The spatial distribution of the mean fluorescence lifetime ( $\tau$ ) at each pixel is shown for wildtype (wt H2B-2FPs cells) (top) or set2(bn129) нав-2FРs mutant animals (bottom). Fluorescence lifetime values ranging between 2.2-3 ns are represented using a continuous pseudo-color scale. Scale bars, $10 \mu \mathrm{m}$. (B) Statistical analysis of the FRET efficiency relative to control (wt), presented as box-and-whisker plots. The mean FRET value is indicated by a cross in each box. ${ }^{* * * *} \mathrm{p}<0.0001$ (two-tailed unpaired $\mathrm{t}$ test). (C) Relative fraction of FRET populations (sub-low, low, intermediate, and high) as defined previously (43) from wt and set2(bn129) pachytene nuclei. ${ }^{*} \mathrm{p}<0.05$; (two-tailed unpaired t test); n.s, non-significant. $\mathrm{n}=5$ gonads (approx. 350 nuclei) for wt, $\mathrm{n}=6$ gonads (approx. 430 nuclei) for set-2(bn129).

\section{Figure 2. set-2 inactivation enhances condensin-II depletion phenotypes. (A) C. elegans condensin} subunits and their vertebrate homologs. (B) Confocal images of distal germline region from wildtype and set-2(bn129) animals treated with empty vector or kle-2 RNAi. Representative images show examples of "wildtype-like" and "strong" phenotypes, with their presence indicated as percentage (\%) of total ( $\mathrm{n}=900$, from 9 independent biological replicates) (scale bar, $20 \mu \mathrm{m}$ ). Arrow indicates the presence of chromatin bridge (scale bar, $10 \mu \mathrm{m}$ ). Images correspond to a Max intensity projection using 
Fiji. (C) kle-2 and capg-1 mRNA levels in wildtype and set-2(bn129) mutant animals after RNAi

880 directed against the respective genes. Relative fold change was calculated with respect to empty vector 881 condition, following normalization with $p m p-3$ and $c d c-42 .\left[^{*}\right] \mathrm{p}<0.05$ t-test. (D) Percentage of 882 germlines with "strong" phenotype after RNAi directed against condensin-II (smc-4, capg-2, kle-2 and 883 hcp-6), condensins-I (capg-1, dpy-28) and condensin-IDC (dpy-27) in wildtype or set-2(bn129) mutants. $884 \mathrm{n}=$ number of animals scored from 9 independent experiments for $s m c-4$ and $k l e-2,4$ for $h c p-6$, and 5 885 for capg- 1 and capg-2, and 2 for $d p y-28$ and $d p y-27$. All scoring was performed in blind. [***] $\mathrm{p}<0.001$ 886 (t-test).

Figure 3. Enhancement of $h c p-6(m r 17)$ phenotypes in set-2(bn129) mutant animals. (A) Schematic diagram of HCP-6 protein and position of the mr17 mutation. Conserved ARM/HEAT and Cnd1 890 (Condensin complex subunit 1) domains are highlighted in red. (B) Brood size per animals at indicated 891 temperatures $\left(15^{\circ} \mathrm{C}, 20^{\circ}, 25^{\circ} \mathrm{C}\right),\left(\mathrm{n}=11\right.$ animals per genotypes at $15^{\circ} \mathrm{C}$ and $25^{\circ} \mathrm{C}$, and 33 at $\left.20^{\circ} \mathrm{C}\right)$. [***] $892 \mathrm{p}<0.001,\left[{ }^{*}\right]<0.05$ (t-test adjusted for multiple comparison with the Bonferroni method). (C) 893 Representative confocal images of germlines stained by orange acridine. Asterisks indicate apoptotic 894 cells. (D) quantification of the number of apoptotic cells in the germline of animals switched at $20^{\circ} \mathrm{C}$ for $24 \mathrm{~h}$ ( $\mathrm{n}>20$ gonads per genotypes). A Wilcoxon test was performed after a significant differences with a Kruskal Wallis test, [ns] non significative difference, $\left[{ }^{*}\right] \mathrm{p}<0.05,[* * *] \mathrm{p}<0.001,\left[{ }^{* * * *}\right] \mathrm{p}<0.0001$.

897

898 Figure 4. Enhancement of the top-2 mutant phenotype in absence of set-2. (A) Representative 899 images of DAPI stained adult germlines showing different phenotypic classes. (Scale bar, $10 \mu \mathrm{m})$. (B) 900 Scoring of phenotypic classes. Animals were shifted to $24^{\circ} \mathrm{C}$ at the $\mathrm{L} 1$ stage and allowed to develop to 901 adulthood. Germlines categories are as defined in Materials and Methods. ${ }^{* * * *} \mathrm{p}<0.0001, * * * \mathrm{p}<0.001$ 902 significant difference between mutant backgrounds using chi-square test and FDR correction (ns: non903 significant). Scale bar, $50 \mu \mathrm{m}$. (C) Enlargement of pachytene and diakinetic nuclei from wildtype or 904 mutant animals. For top-2 single and top-2;set-2 double mutants, representative nuclei from normal 905 (left) and short (right) germlines are shown. Nuclei containing more than 6 DAPI stained bodies were 
observed in both "short" and "normal" germlines. (D) Scoring of aneuploid nuclei from cells in

907 diakinesis (Scale bar, $10 \mu \mathrm{m})$.

908

909

Figure 5. Inactivation of COMPASS targeting component $c f p-1$ mimics set-2 inactivation. (A)

910 Percentage of germlines with "strong" phenotype after RNAi directed against condensin-II (smc-4 or

911 kle-2) in wildtype, $c f p-1(\operatorname{tm} 6369)$ and $r b r-2(t m 1231)$ mutants. $\mathrm{n}=$ number of animals scored from at

912 least 3 independent experiments. $\mathrm{p}<0.05$, [***] $\mathrm{p}<0.001$, [****] $\mathrm{p}<0.0001$ (t-test); ns, not significant.

913 (B) Decreased chromatin compaction in $c f p-1$ mutant germlines. Spatial distribution of the mean

914 fluorescence lifetime is shown for wildtype (wt H2B-2FPs) or $c f p-1$ H2B-2FPs mutant animals. Fluorescence

915 lifetime values $(\tau)$ ranging from $2.0 \mathrm{~ns}$ to $2.8 \mathrm{~ns}$ are represented using a continuous pseudo-color scale.

916 Scale bars, $10 \mu \mathrm{m}$. Statistical analysis of the FRET efficiency relative to control wt is presented as box-

917 and-whisker plots. Mean FRET value is indicated by a cross in each box. ${ }^{*} \mathrm{p}<0.05$ (two-tailed unpaired

$918 \mathrm{t}$ test). $\mathrm{n}=13$ gonads for $\mathrm{wt}, \mathrm{n}=11$ gonads for $c f p-1$.

919

920

Figure 6. Working model for cooperation between COMPASS, condensin-II and TOP-2 in

921 chromosome organization. In wild type, proper chromosome compaction results from the activity of

922 condensin-I (connected yellow circles), condensin-II (connected orange circles) and TOP-2 (blue broken

923 ring). The concerted action of condensins results in the formation of arrays of helical loops, with

924 condensin-II generating outer loops and condensin-I forming inner loops (80). TOP-2 may contribute to

925 compaction by modulating chromatin loops (60), or actively introducing self-entanglement in DNA

926 (32). COMPASS may mediate interactions between loops, possibly by contributing to the clustering of

927 transcribed loci (110). The absence of COMPASS results in subtle defects in chromosome organization

928 (weakly disorganized chromatin), but overall chromosome architecture is maintained by the action of

929 condensins, TOP-2 and additional proteins. Partial depletion of condensin-II or TOP-2 in the absence of

930 COMPASS results in cumulative defects in chromosome organization. 


\section{Supplementary data}

\section{H3K4me3 Immunostaining}

934 Immunostaining was as previously described (25). Z-stack images of gonads were acquired using a Zeiss

935 LSM710 inverted confocal microscope with 40X oil Immersion objective. Z-stack of germlines were 936 acquired every $0.25 \mu \mathrm{m}$, images correspond to a projection using Max intensity method and Fiji macros.

937 Antisera and the dilutions used were as followed: rabbit anti H3K4me3 (Diagenode, 15310003[CS938 003100]; 1:12000) and anti-rabbit Alexa Fluor 555 (Invitrogen/Molecular probes \#A21428; 1:1000).

\section{Scoring condensin I RNAi animals}

940 For scoring the efficacy of RNAi, wildtype or set-2(bn129) L4 stage animals were transferred to the 941 same RNAi plates used for scoring germline phenotypes and allowed to develop into adults at $20^{\circ} \mathrm{C}$.

942 Following $24 \mathrm{hrs}$ of egg laying, animals were removed and the body length of F1 progeny measured 3 943 days later, at the L4 stage (based on vulval morphology). Animals were placed on $4 \%$ agar pads in $1 \mathrm{X}$

944 M9 complemented with 10mM Levamisole, and observed by DIC on an Axio Imager A2 microscope 945 and EC plan Neofluar 10X/0.5 objective. Length of individual worms was measured using Fiji macros.

\section{Scoring embryonic viability}

947 Between 10 to $20 \mathrm{~L} 4$ hermaphrodites grown at the permissive temperature $\left(15^{\circ} \mathrm{C}\right)$, were placed on NGM 948 plates at the restrictive temperature of $20^{\circ} \mathrm{C}$ during $24 \mathrm{~h}$, then removed. Eggs laid were allowed to 949 develop during $24 \mathrm{~h}$ at $20^{\circ} \mathrm{C}$ (Normal development takes $18 \mathrm{~h}$ at $20^{\circ} \mathrm{C}$ from fertilization to hatching). 950 Then dead eggs and larvae were recovered in 1X M9 and washed twice in 1X M9 in order to remove 951 bacteria. Eggs and larvae were placed on $4 \%$ agar pads and a coverslip was placed on top. The stage of 952 embryos was determined by DIC observation with EC Plan-Neofluar 100X/1.3 oil objective and 953 AxioImager A2 (Zeiss). At least 75 embryos were scored per genotypes, and the experiment repeated 3 954 independent times.

\section{RNA sequencing of dissected gonads}

956 Gonad dissections and extractions were performed as in (25). Briefly, prior to dissection worms were 957 placed on NGM plates without food to expel bacteria from the gut. Gonads of 5 to 7 young adults at the 
Extruded gonads were cut at the elbow and the distal part recovered using a drawn capillary and transferred to $30 \mu 1$ of XB extraction buffer (Kit Picopure, Life technology, \# 12204-01), frozen in liquid nitrogen and stored at $-80^{\circ} \mathrm{C}$. For RNA preparation tubes were thawed, the volume of XB extraction buffer adjusted to $100 \mu$, and RNA purified using the PicoPure kit (Life Technology, \# 12204-01) according to the manufacturer's instructions. Elution was in $13 \mu \mathrm{l}$ of nuclease-free water. The integrity of RNA was evaluated using Tape Station 4200 (Agilent), and the concentration of RNA measured using platform (IGBMC, Strasbourg, France), and sequencing by an Illumina Hiseq 4000 device. Bioinformatic analysis was carried out under Galaxy (126). Sequence reads were mapped onto the reference genome (WS254) with the RNA-STAR tool (Version 2.4.1d). Sequences with a quality of cartography lower than 10 were removed with SAMtools (Version0.1.19). The expression level of each

971 gene for each sample was calculated with htseq-count (Version 0.7.2). Differential analysis of gene 972 expression between the different strains was carried out with the DESeq2 package version 1.16.1 (127) 973 under R version 3.4.4. Additional analyzes were performed with R.

Table S1. Genes misregulated in dissected gonads from set-2 mutant animals

Figure S1. set-2 inactivation differentially impacts H3K4me3 in the germline (A) Z-projection of 978 confocal images through the mitotic region, early-mid pachytene and late pachytene nuclei, and 979 diakinesis. Gonads were dissected, fixed and probed with rabbit anti-H3K4me3 antibodies (Diagenode, \#15310003) and counter-stained with DAPI. Images were taken with the same laser parameter for each condition. (Scale bar, $10 \mu \mathrm{m}$ ). (B) Confocal images of DAPI stained germlines of fertile wildtype 982 animals and sterile set-2(bn129) after 4 generations at $25^{\circ} \mathrm{C}$. (Scale bar, $\left.10 \mu \mathrm{m}\right)$.

984 Figure S2. Tagged histones H2B are correctly expressed and incorporated into chromatin in set-2 985 mutants. (A) Mean fluorescence intensity of fluorescently-tagged H2B histones (GFP-H2B and 
mCherry-H2B) from wt and set-2(bn129) mutant pachytene cells. Each data point shows the mean

987 fluorescence intensity \pm S.D. from 8 and 12 gonads representing 549 and 698 nuclei from wt and set-

988

989

990

991

992

993

994

995

996

997

998

999

1000

1001

1002

1003

1004

1005

1006

1007

1008

1009

1010

1011

1012

2(bn129), respectively. (B) Normalized fluorescence intensity recovery of GFP-H2B from wt (black squares) and set-2(bn129) (red squares) pachytene cells after laser photo-bleaching (FRAP experiments). Each data point shows the mean \pm S.D. for 11 and 8 pachytene cells from wt and set2(bn129), respectively.

Figure S3. Polyploid cells resulting from kle-2 knockdown in set-2 mutant germlines. Wildtype diploid cells carrying a GFP LacO cassette integrated on chromosome IV and expressing lacI show 2 GFP spots/nucleus. Polyploid nuclei from kle-2(RNAi);set-2 germlines showing a strong phenotype are larger and show more than two GFP spots (circled in white) (Scale bar, $10 \mu \mathrm{m}$ ).

Figure S4. Condensin I depletion in wildtype and set-2 mutant animals results in similar reduction in body size. Condensin I knock down animals are smaller in length than control empty vector (dpy phenotype (128). A similar decrease in body size was observed in wildtype and set-2 mutants. [****] p $<0.0001$ (t-test). ns, not significant. For wild type: empty vector n=32, capg- 1 RNAi n=23, dpy-27 RNAi $\mathrm{n}=29, d p y-28$ RNAi $\mathrm{n}=30$; for set-2(bn129): empty vector $\mathrm{n}=31$, capg- 1 RNAi $\mathrm{n}=26, d p y-27$ RNAi $\mathrm{n}=30, d p y-28$ RNAi $\mathrm{n}=26$

Figure S5. Germline defects of $h c p-6(m r 17)$ mutant animals and suppression of $h c p-6(m r 17)$ embryonic lethality. (A) $h c p-6(m r 17)$ gonads are highly disorganized. Young adult wildtype, set-2, $h c p$ 6 and set-2;hpc-6 mutant animals shifted to $25^{\circ} \mathrm{C}$ at the L4 stage were DAPI stained and observed on AxioImager A2 (Zeiss). (B) set-2 partially suppresses the embryonic lethality of $h c p-6(m r 17)$ mutants. Percentage of embryonic lethality at $\left.20^{\circ} \mathrm{C} .{ }^{*}\right] \mathrm{p}<0.05$ (t-test). number of progeny scored for wildtype, set-2(bn129), hcp-6(mr17) and hcp-6(mr17);set-2(bn129) mutants was 10109, 8227, 8757 and 3961, respectively. 3 independent biological replicates were performed for each genotype. 
1013 Figure S6. Loss of H3K4me3 in $c f p-1$ mutant germlne and efficacy and scoring of $c f p-1$ and $r b r-2$

1014 RNAi in independent experiments. (A) decreased H3K4me3 in $c f p-1$ (tm6369) mutant germline. (B)

1015 kle-2 mRNA levels in wt, $c f p-1(t m 6369)$ and $r b r-2(t m 1231)$ mutant animals. For each of the three

1016 independent experiments, relative fold change was calculated with respect to empty vector condition,

1017 following normalization with $p m p-3$ and $c d c-42$. (C) Percentage of germlines with "strong" phenotype

1018 in each of the three replicas after RNAi directed against kle-2 RNAi. Note that although the efficacy of

1019 kle-2 mRNA knockdown varied between the three experiments, the \% of abnormal germlines scored

1020 for each genotype (wt, $c f p-1$ and $r b r$-2) was highly similar. 


\section{Figures}

A

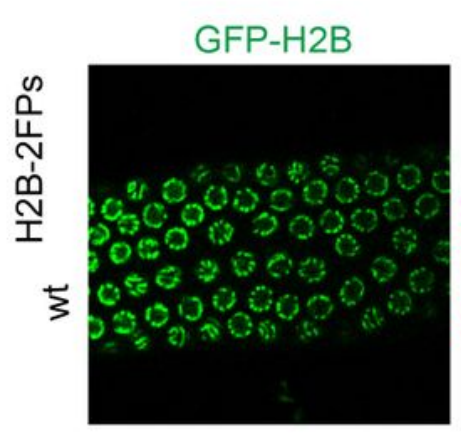

mCherry-H2B

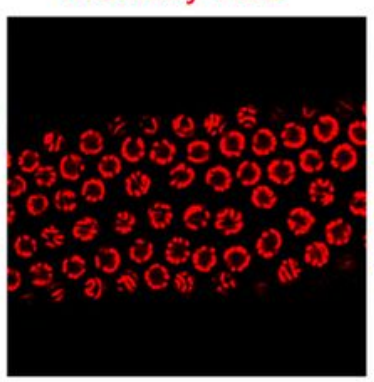

fluorescence
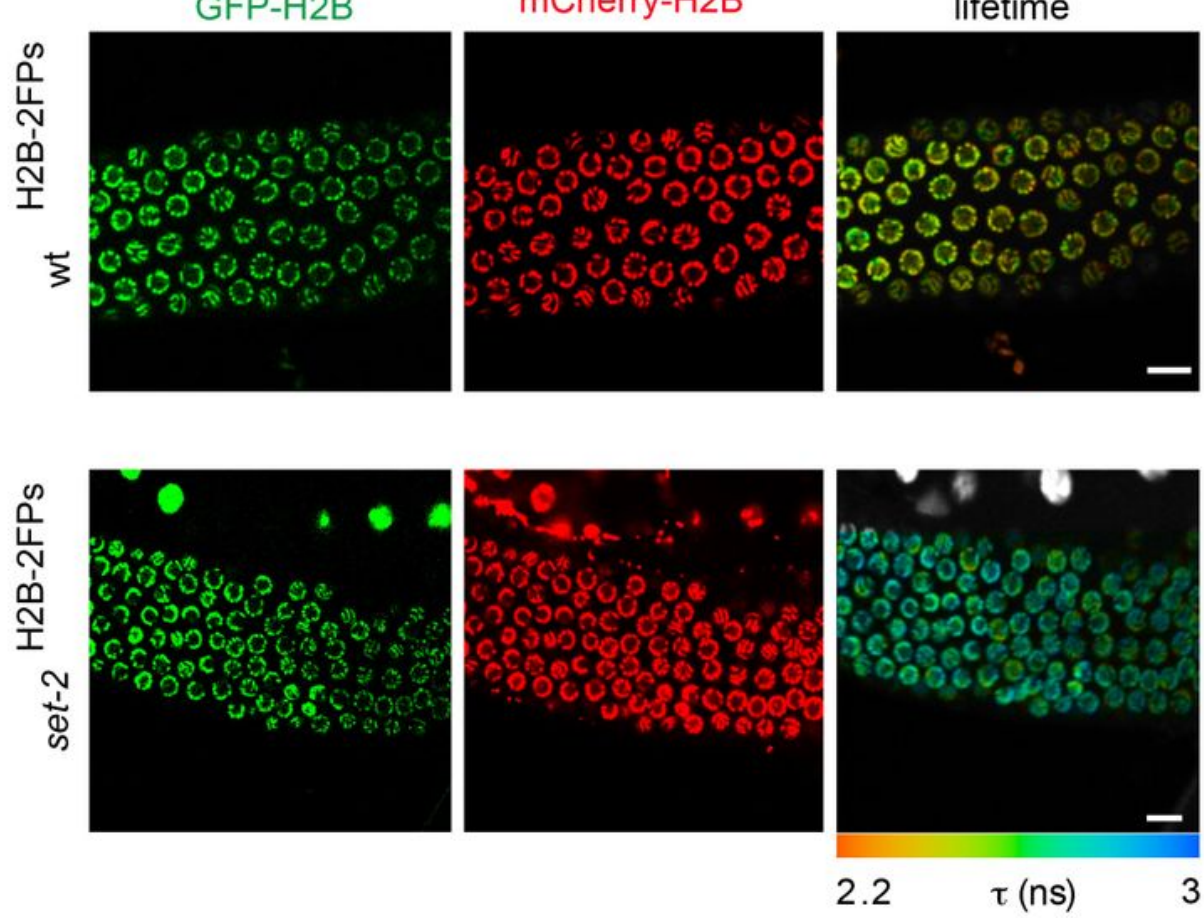

B

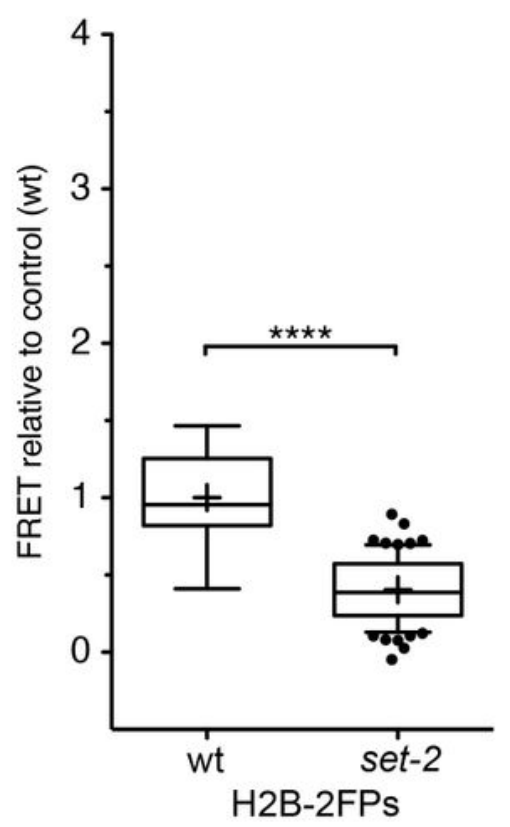

C

FRET efficiency

$\square$ sub-low (0-10\%)

$\square$ low (10-30\%)

$\square$ intermediate (30-60\%)

high (60-80\%)

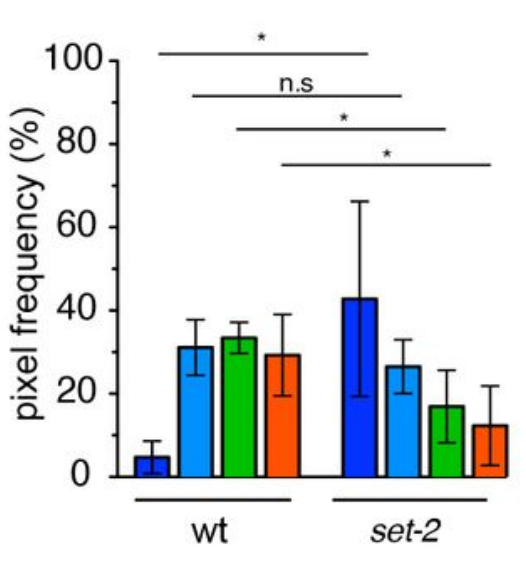

Figure 1

set-2 inactivation influences nanoscale chromatin compaction in the germline. (A) Fluorescence intensities of GFP-H2B (green) and mCherry-H2B (red) from pachytene-stage germ cells expressing H2B2FPs. FLIM (right) images of H2B-2FPs pachytene-stage cells. The spatial distribution of the mean 
fluorescence lifetime ( $\square$ ) at each pixel is shown for wildtype (wt H2B-2FPs cells) (top) or set- 2(bn129) H2B-2FPs mutant animals (bottom). Fluorescence lifetime values ranging between 2.2-3 ns are represented using a continuous pseudo-color scale. Scale bars, $10 \mathrm{Dm}$. (B) Statistical analysis of the FRET efficiency relative to control (wt), presented as box-and-whisker plots. The mean FRET value is indicated by a cross in each box. ${ }^{* \star \star *} \mathrm{p}<0.0001$ (two-tailed unpaired $t$ test). (C) Relative fraction of FRET populations (sub-low, low, intermediate, and high) as defined previously (43) from wt and set- 2(bn129) pachytene nuclei. ${ }^{*} \mathrm{p}<0.05$; (two-tailed unpaired $\mathrm{t}$ test); $\mathrm{n} . \mathrm{s}$, non-significant. $\mathrm{n}=5$ gonads (approx. 350 nuclei) for wt, $n=6$ gonads (approx. 430 nuclei) for set-2(bn129). 
A

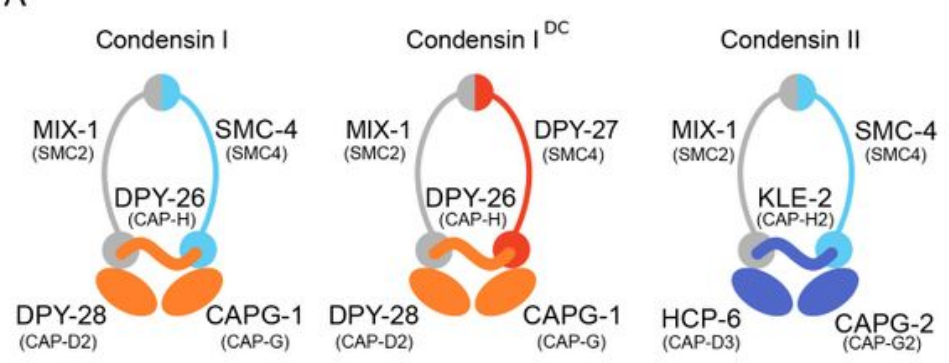

B
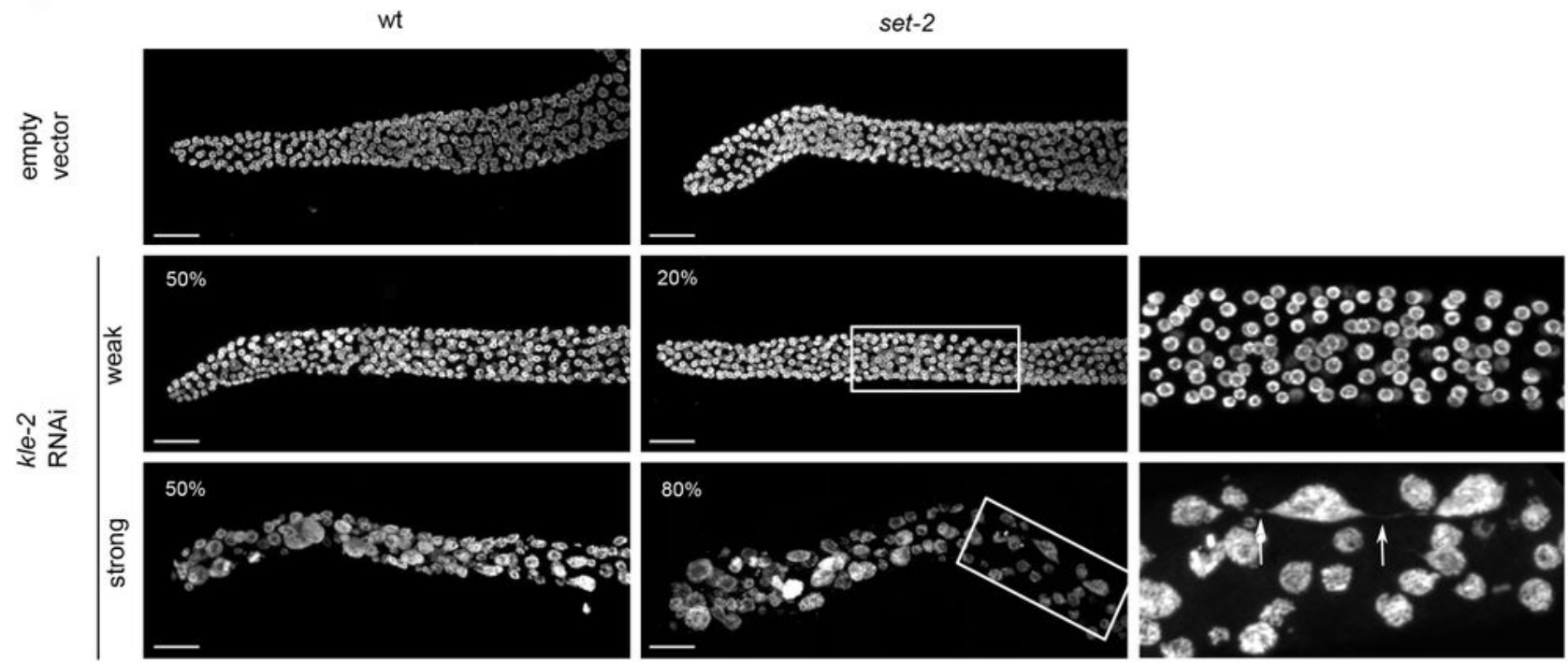

C
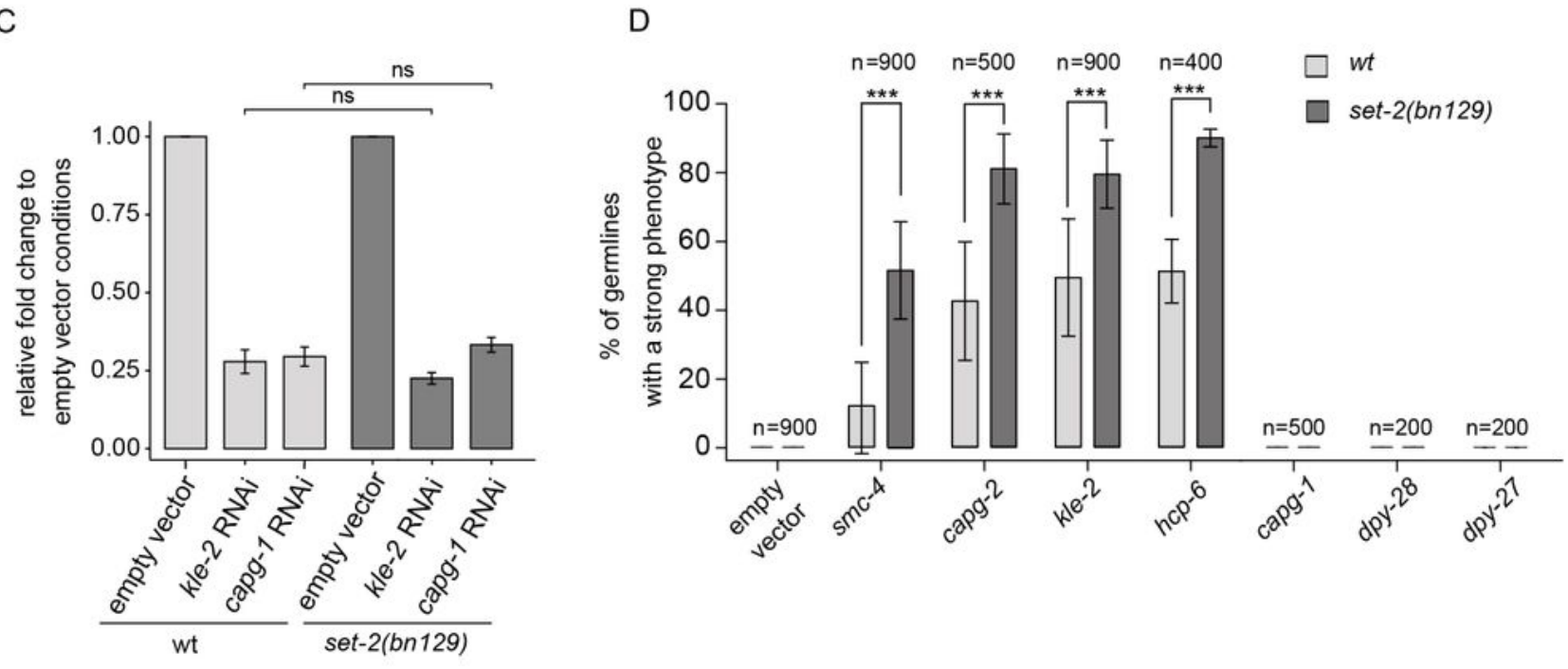

Figure 2

873 Figure 2. set-2 inactivation enhances condensin-II depletion phenotypes. (A) C. elegans condensin subunits and their vertebrate homologs. (B) Confocal images of distal germline region from wildtype and set-2(bn129) animals treated with empty vector or kle-2 RNAi. Representative images show examples of "wildtype-like" and "strong" phenotypes, with their presence indicated as percentage (\%) of total ( $n=900$, from 9 independent biological replicates) (scale bar, $20 \mu \mathrm{m}$ ). Arrow indicates the presence of chromatin 
bridge (scale bar, $10 \mu \mathrm{m}$ ). Images correspond to a Max intensity projection using Fiji. (C) kle-2 and capg-1 mRNA levels in wildtype and set-2(bn129) mutant animals after RNAi 879 directed against the respective genes. Relative fold change was calculated with respect to empty vector 880 condition, following normalization with pmp-3 and cdc-42. [*] p<0.05 t-test. (D) Percentage of 881 germlines with "strong" phenotype after RNAi directed against condensin-II (smc-4, capg-2, kle-2 and 882 hcp-6), condensins-I (capg-1, dpy-28) and condensin-IDC (dpy-27) in wildtype or set-2(bn129) mutants. $883 \mathrm{n}=$ number of animals scored from 9 independent experiments for smc- 4 and kle-2, 4 for hcp-6, and 5884 for capg- 1

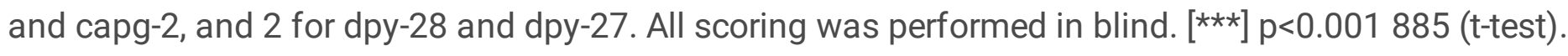


A

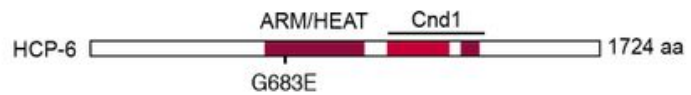

B

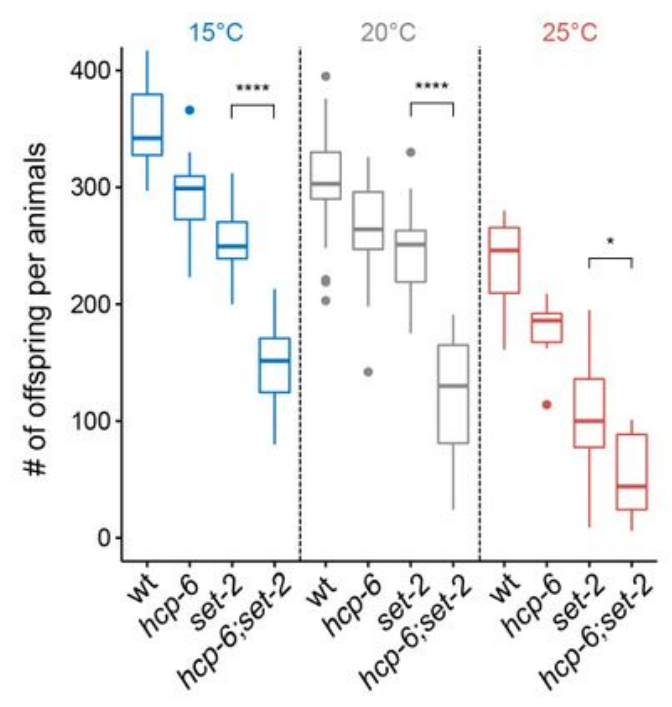

C

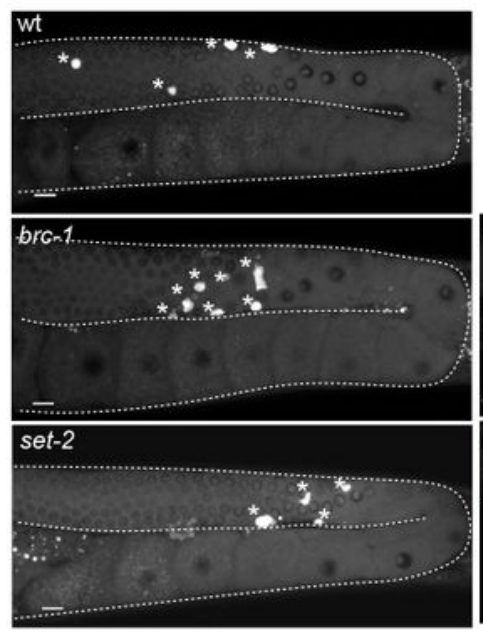

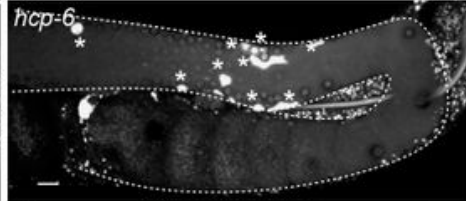

hcp-6;set-2

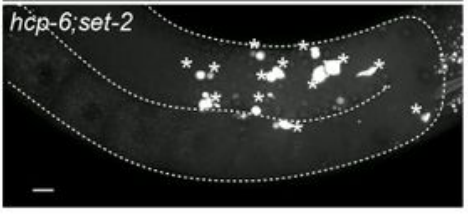

D

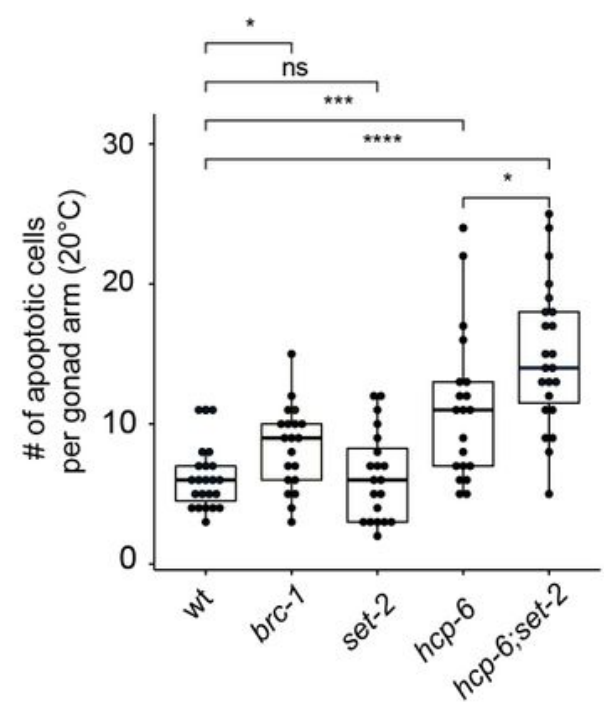

\section{Figure 3}

888 Figure 3. Enhancement of hcp-6(mr17) phenotypes in set-2(bn129) mutant animals. (A) Schematic 889 diagram of HCP-6 protein and position of the mr17 mutation. Conserved ARM/HEAT and Cnd1 890 (Condensin complex subunit 1) domains are highlighted in red. (B) Brood size per animals at indicated

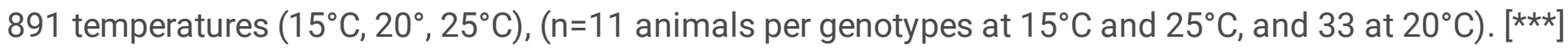
$892 p<0.001,[*]<0.05$ (t-test adjusted for multiple comparison with the Bonferroni method). (C) 893 
Representative confocal images of germlines stained by orange acridine. Asterisks indicate apoptotic 894 cells. (D) quantification of the number of apoptotic cells in the germline of animals switched at $20^{\circ} \mathrm{C} 895$ for $24 \mathrm{~h}$ ( $\mathrm{n}>20$ gonads per genotypes). A Wilcoxon test was performed after a significant differences with 896 a Kruskal Wallis test, [ns] non significative difference, $[*] p<0.05$, $[\star \star \star] p<0.001$, $[\star \star \star \star *] p<0.0001$.
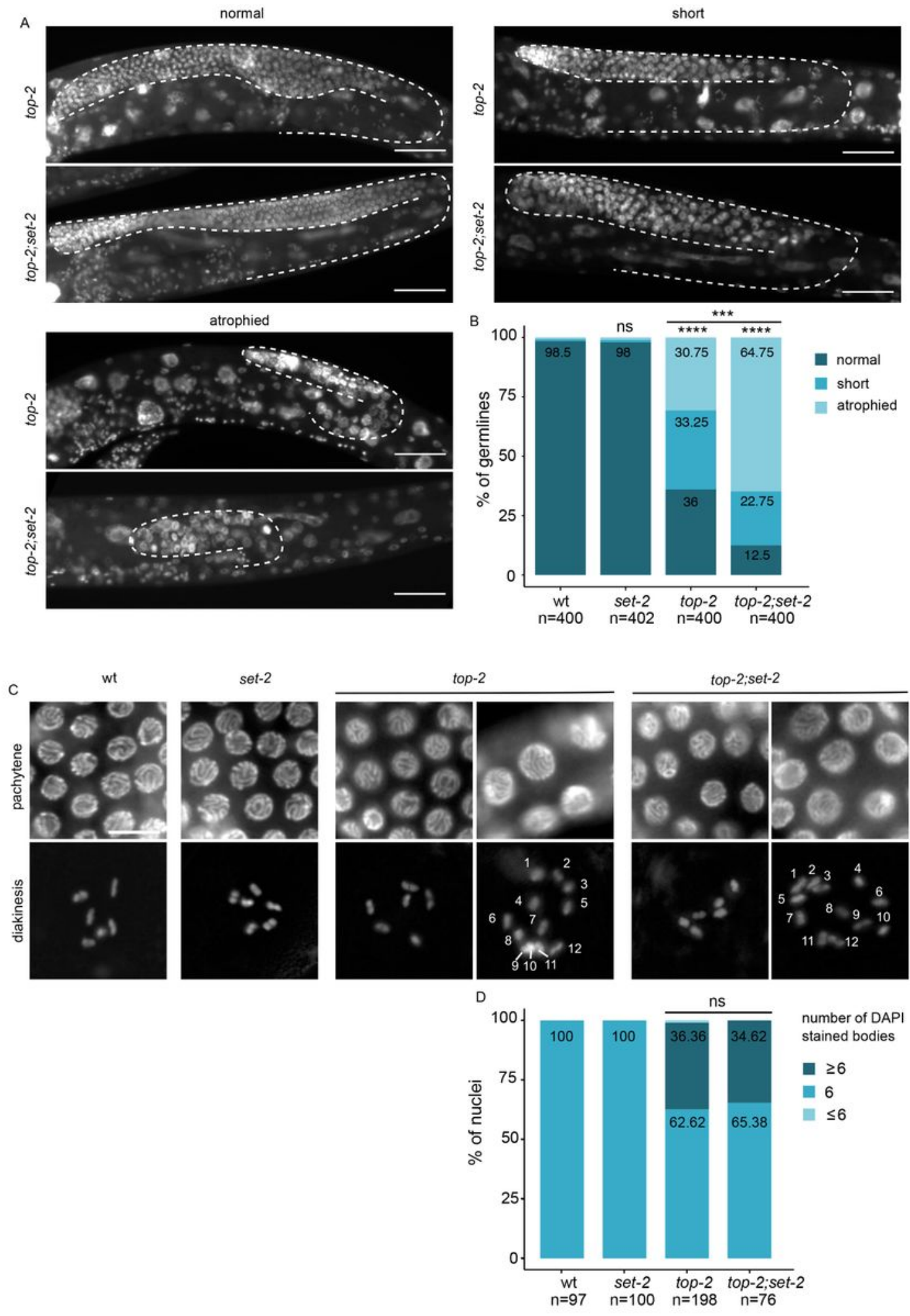

Figure 4 
898 Figure 4. Enhancement of the top-2 mutant phenotype in absence of set-2. (A) Representative 899 images of DAPI stained adult germlines showing different phenotypic classes. (Scale bar, $10 \mu \mathrm{m}$ ). (B) 900 Scoring of phenotypic classes. Animals were shifted to $24^{\circ} \mathrm{C}$ at the L1 stage and allowed to develop to 901 adulthood. Germlines categories are as defined in Materials and Methods. ${ }^{\star \star \star *} p<0.0001,{ }^{\star \star \star} p<0.001$ 902 significant difference between mutant backgrounds using chi-square test and FDR correction (ns: non- 903 significant). Scale bar, $50 \mu \mathrm{m}$. (C) Enlargement of pachytene and diakinetic nuclei from wildtype or 904 mutant animals. For top-2 single and top-2;set-2 double mutants, representative nuclei from normal 905 (left) and short (right) germlines are shown. Nuclei containing more than 6 DAPI stained bodies were 30906 observed in both "short" and "normal" germlines. (D) Scoring of aneuploid nuclei from cells in 907 diakinesis (Scale bar, $10 \mu \mathrm{m}$ ). 


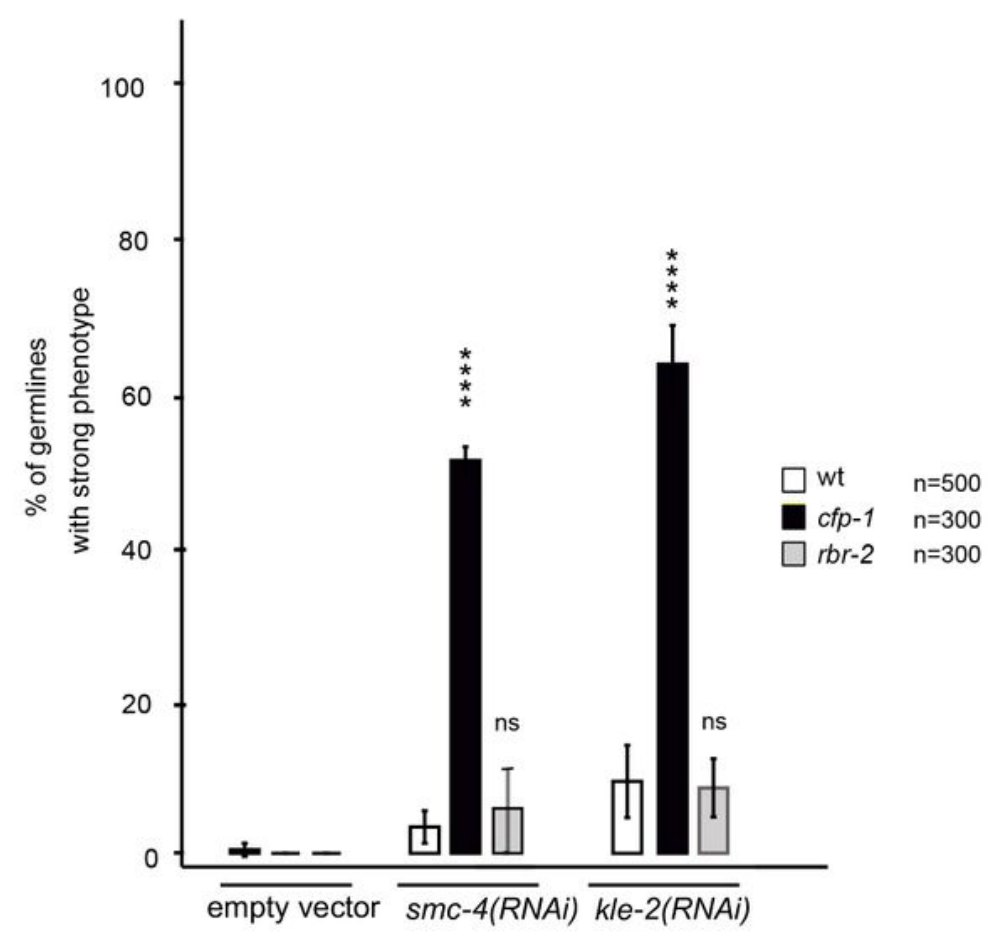

B

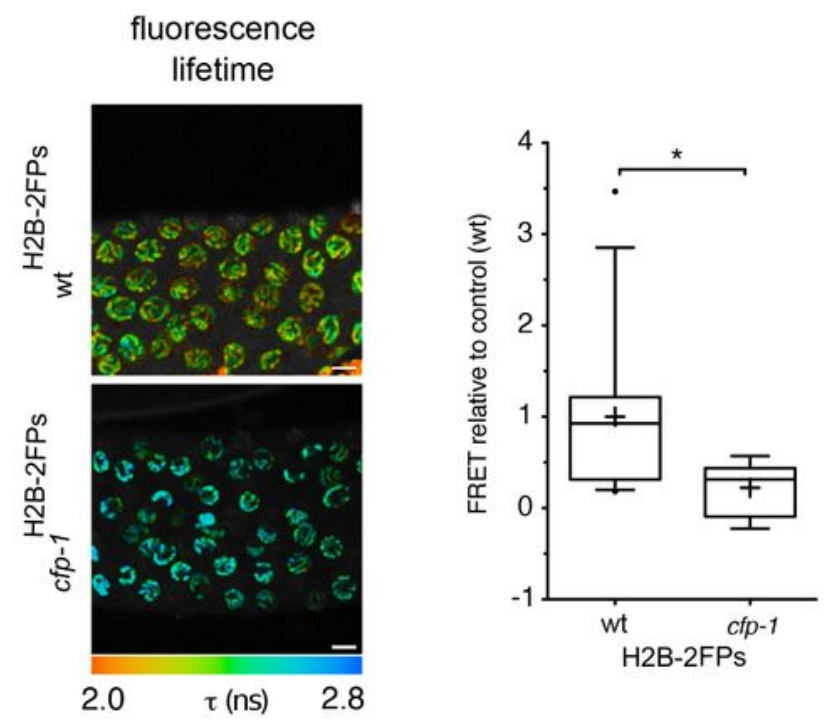

Figure 5

. Inactivation of COMPASS targeting component cfp-1 mimics set-2 inactivation. (A) 910 Percentage of germlines with "strong" phenotype after RNAi directed against condensin-II (smc-4 or 911 kle-2) in wildtype, cfp-1 (tm6369) and rbr-2(tm1231) mutants. $n=$ number of animals scored from at 912 least 3

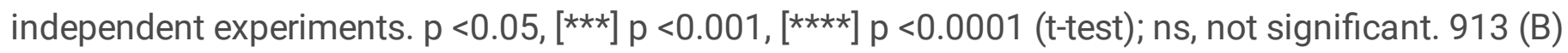
Decreased chromatin compaction in cfp-1 mutant germlines. Spatial distribution of the mean 914 
fluorescence lifetime is shown for wildtype (wt H2B-2FPs) or cfp-1 H2B-2FPs mutant animals.

Fluorescence 915 lifetime values $(\square)$ ranging from 2.0 ns to $2.8 \mathrm{~ns}$ are represented using a continuous pseudo-color scale. 916 Scale bars, $10 \mathrm{Dm}$. Statistical analysis of the FRET efficiency relative to control wt is presented as box- 917 and-whisker plots. Mean FRET value is indicated by a cross in each box. ${ }^{*}<$ 0.05 (two-tailed unpaired $918 \mathrm{t}$ test). $\mathrm{n}=13$ gonads for wt, $\mathrm{n}=11$ gonads for cfp- 1 .

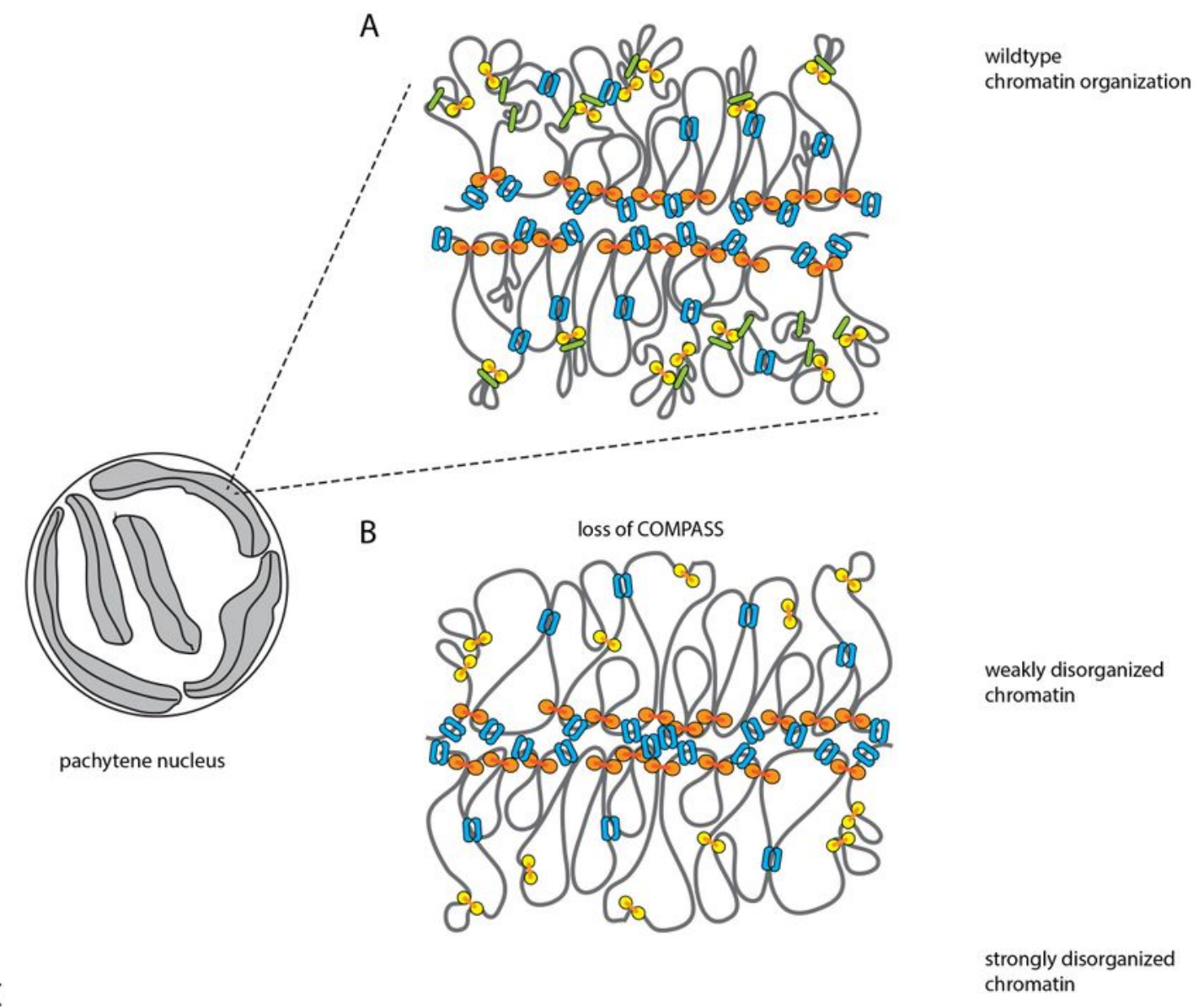

C

Ioss of COMPASS and depletion of condensin-II

loss of COMPASS and depletion of TOP-2
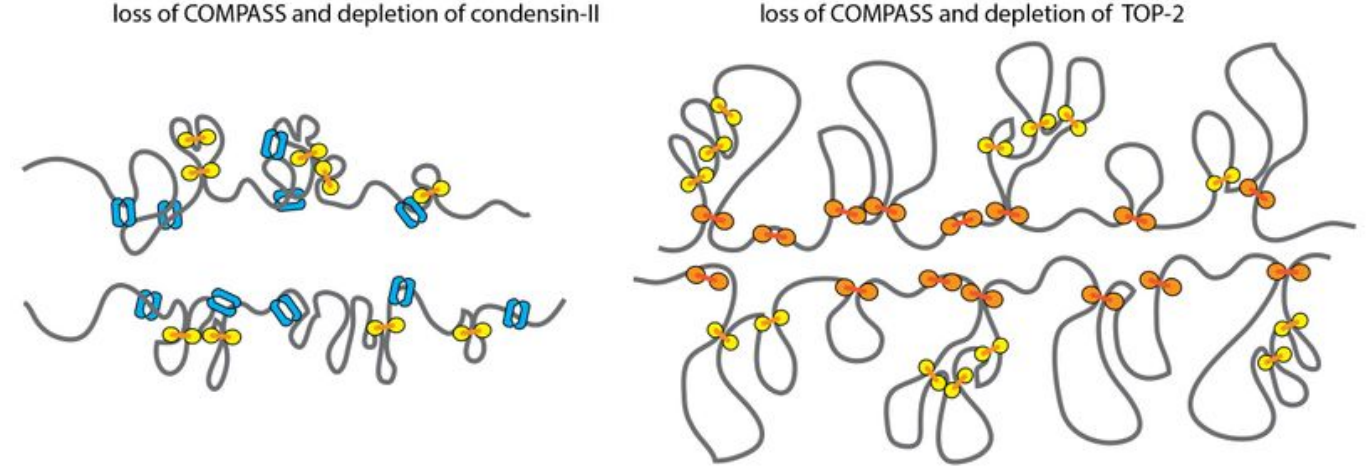

0 cOMPASS
TOP-2

\section{Figure 6}


. Working model for cooperation between COMPASS, condensin-II and TOP-2 in 921 chromosome organization. In wild type, proper chromosome compaction results from the activity of 922 condensin-I (connected yellow circles), condensin-II (connected orange circles) and TOP-2 (blue broken 923 ring). The concerted action of condensins results in the formation of arrays of helical loops, with 924 condensin-II generating outer loops and condensin-I forming inner loops (80). TOP-2 may contribute to 925 compaction by modulating chromatin loops (60), or actively introducing self-entanglement in DNA 926 (32). COMPASS may mediate interactions between loops, possibly by contributing to the clustering of 927 transcribed loci (110). The absence of COMPASS results in subtle defects in chromosome organization 928 (weakly disorganized chromatin), but overall chromosome architecture is maintained by the action of 929 condensins, TOP-2 and additional proteins. Partial depletion of condensin-II or TOP-2 in the absence of 930 COMPASS results in cumulative defects in chromosome organization.

\section{Supplementary Files}

This is a list of supplementary files associated with this preprint. Click to download.

- Tables1.xls

- FiguresS1S5.pdf 